\title{
Geometry of Control-Affine Systems
}

\author{
Jeanne N. CLELLAND ${ }^{\dagger}$, Christopher G. MOSELEY ${ }^{\ddagger}$ and George R. WILKENS ${ }^{\S}$ \\ $\dagger$ Department of Mathematics, 395 UCB, University of Colorado, Boulder, CO 80309-0395, USA \\ E-mail: Jeanne.Clelland@colorado.edu \\ $\ddagger$ Department of Mathematics and Statistics, Calvin College, Grand Rapids, MI 49546, USA \\ E-mail: cgm3@calvin.edu \\ $\S$ Department of Mathematics, University of Hawaii at Manoa, 2565 McCarthy Mall, \\ Honolulu, HI 96822-2273, USA \\ E-mail: grw@math.hawaii.edu
}

Received April 02, 2009, in final form September 28, 2009; Published online October 07, 2009 doi:10.3842/SIGMA.2009.095

\begin{abstract}
Motivated by control-affine systems in optimal control theory, we introduce the notion of a point-affine distribution on a manifold $\mathcal{X}$ - i.e., an affine distribution $\mathcal{F}$ together with a distinguished vector field contained in $\mathcal{F}$. We compute local invariants for point-affine distributions of constant type when $\operatorname{dim}(\mathcal{X})=n, \operatorname{rank}(\mathcal{F})=n-1$, and when $\operatorname{dim}(X)=3, \operatorname{rank}(\mathcal{F})=1$. Unlike linear distributions, which are characterized by integervalued invariants - namely, the rank and growth vector - when $\operatorname{dim}(X) \leq 4$, we find local invariants depending on arbitrary functions even for rank 1 point-affine distributions on manifolds of dimension 2 .
\end{abstract}

Key words: affine distributions; control theory; exterior differential systems; Cartan's method of equivalence

2000 Mathematics Subject Classification: 58A30; 53C17; 58A15; 53C10

\section{Introduction}

In [8] and [9], we introduced the notion of sub-Finsler geometry as a natural geometric setting for certain problems in optimal control theory. In local coordinates, a control system may be represented as an underdetermined system of ordinary differential equations of the form

$$
\dot{x}=f(x, u),
$$

where $x \in \mathbb{R}^{n}$ represents the state vector of the system and $u \in \mathbb{R}^{s}$ represents the control vector, i.e., variables which may be specified freely in order to "steer" the system in a desired direction. More generally, $x$ and $u$ may take values in an $n$-dimensional manifold $X$ and an $s$-dimensional manifold $\mathcal{U}$, respectively. Typically there are constraints on how the system may be "steered" from one state to another, so that $s<n$. The systems of greatest interest are controllable, i.e., given any two states $x_{1}, x_{2}$, there exists a solution curve of (1.1) connecting $x_{1}$ to $x_{2}$.

The control systems for which sub-Finsler (or sub-Riemannian) geometry are most relevant are control-linear systems. These are systems for which the right-hand side of (1.1) is linear in the control variables $u$ and depends smoothly on the state variables $x$; i.e., systems of the form

$$
\dot{x}=A(x) u,
$$

where $A(x)$ is an $n \times s$ matrix whose entries are smooth functions of $x$. For such a system, admissible paths in the state space are those for which the tangent vector to the path at each

\footnotetext{
*This paper is a contribution to the Special Issue "Élie Cartan and Differential Geometry". The full collection is available at http://www.emis.de/journals/SIGMA/Cartan.html
} 
point $x \in \mathcal{X}$ is contained in the subspace $\mathcal{D}_{x} \subset T_{x} \mathcal{X}$ determined by the image of the matrix $A(x)$. If this matrix has constant (maximal) rank $s$ on $\mathcal{X}$, then $\mathcal{D}$ is a rank $s$ linear distribution ${ }^{1}$ on $X$. Thus the admissible paths in the state space are precisely the horizontal curves of the distribution $\mathcal{D}$, i.e., curves whose tangent vectors at each point are contained in $\mathcal{D}$.

A sub-Finsler (resp., sub-Riemannian) metric on the distribution $(X, \mathcal{D})$ is defined by specifying a Finsler (resp., Riemannian) metric on each of the subspaces $\mathcal{D}_{x}$. Such a metric may represent a cost function for the control problem, and geodesics for the metric may be thought of as stationary trajectories for the corresponding optimal control problem.

While the class (1.2) of control-linear systems contains many interesting examples, a much broader class of interest is that of control-affine systems, i.e., systems of the form

$$
\dot{x}=a_{0}(x)+A(x) u,
$$

where $A(x)$ is a smoothly-varying $n \times s$ matrix and $a_{0}(x)$ is a smooth vector field on $X$. (The vector field $a_{0}(x)$ is known as the drift vector field.)

Example 1.1. The class of control-affine systems contains as a sub-class the linear control systems. These are systems of the form

$$
\dot{x}=A x+B u,
$$

where $A$ is a constant $n \times n$ matrix and $B$ is a constant $n \times s$ matrix. Note that despite the terminology, the linear control system (1.4) is not control-linear unless $A=0$.

The geometric structure corresponding to a control-affine system is an affine distribution on the state space $\mathcal{X}$. More precisely (see $[12,13])$ :

Definition 1.2. A rank $s$ affine distribution $\mathcal{F}$ on an $n$-dimensional manifold $\mathcal{X}$ is a smoothlyvarying family of $s$-dimensional, affine linear subspaces $\mathcal{F}_{x} \subset T_{x} X$. We will say that $\mathcal{F}$ is strictly affine if none of the affine subspaces $\mathcal{F}_{x} \subset T_{x} \mathcal{X}$ are linear subspaces. Associated to an affine distribution $\mathcal{F}$ is the direction distribution

$$
L_{\mathcal{F}}=\left\{\xi_{1}-\xi_{2} \mid \xi_{1}, \xi_{2} \in \mathcal{F}\right\}
$$

Note that $L_{\mathcal{F}}$ is a rank $s$ linear distribution on $X$.

In this paper, we will limit our attention to distributions (both linear and affine) which are bracket-generating (or almost bracket-generating in the affine case) and have constant type. In order to make these notions precise, we need the notion of the growth vector of a distribution (cf. [21]). Let $\mathcal{D}$ be a linear distribution on a manifold $\mathcal{X}$, and let $\mathcal{D}$ also denote the sheaf of smooth vector fields on $\mathcal{X}$ which are local sections of $\mathcal{D}$. The iterated Lie brackets of vector fields in $\mathcal{D}$ generate a flag of subsheaves

$$
\mathcal{D}=\mathcal{D}^{1} \subset \mathcal{D}^{2} \subset \cdots \subset T X,
$$

defined by $\mathcal{D}^{1}=\mathcal{D}$, and

$$
\mathcal{D}^{i+1}=\mathcal{D}^{i}+\left[\mathcal{D}, \mathcal{D}^{i}\right], \quad i \geq 1 .
$$

At each point $x \in \mathcal{X}$, this flag of subsheaves gives a flag of subspaces of $T_{x} X$ :

$$
\mathcal{D}_{x} \subset \mathcal{D}_{x}^{2} \subset \cdots \subset T_{x} X
$$

\footnotetext{
${ }^{1}$ We use the adjective "linear" to distinguish the usual notion of a distribution $\mathcal{D}$, where each fiber $\mathcal{D}_{x}$ is a linear subspace of $T_{x} \mathcal{X}$, from that of an affine distribution $\mathcal{F}$. Unless otherwise specified, the word "distribution" will refer to a linear distribution throughout this paper.
} 
Definition 1.3. Let $\mathcal{D}^{\infty}=\cup_{i \geq 1} \mathcal{D}^{i} \subset T X$.

- The smallest integer $r=r(x)$ such that $\mathcal{D}_{x}^{r}=\mathcal{D}_{x}^{\infty}$ is called the step of the distribution at $x$.

- The distribution $\mathcal{D}$ is bracket-generating if $\mathcal{D}^{\infty}=T X$.

- Set $n_{i}(x)=\operatorname{dim} \mathcal{D}_{x}^{i}$. The growth vector of $\mathcal{D}$ at $x$ is the integer list $\left(n_{1}(x), n_{2}(x), \ldots\right.$, $\left.n_{r}(x)\right)$, where $r(x)$ is the step of $\mathcal{D}$ at $x$.

- The distribution $\mathcal{D}$ has constant type if $n_{i}(x)$ is constant on $\mathcal{X}$ for all $i$; i.e., if the growth vector of $\mathcal{D}$ is constant on $X$.

Remark 1.4. When working with distributions of constant type, it is customary to consider only bracket-generating distributions. For if $\mathcal{D}$ is not bracket-generating, then $\mathcal{D}^{\infty}$ is a Frobenius distribution on $\mathcal{X}$. The integral manifolds of $\mathcal{D}^{\infty}$ define a local foliation of $\mathcal{X}$ with the property that any horizontal curve of $\mathcal{D}$ is contained in a single leaf of the foliation, and the restriction of $\mathcal{D}$ to each leaf of the foliation is bracket-generating.

We can define similar notions for affine distributions: let $\mathcal{F}$ be an affine distribution on a manifold $\mathcal{X}$, with direction distribution $L_{\mathcal{F}}$. Let $\mathcal{F}$ also denote the sheaf of smooth vector fields on $\mathcal{X}$ which are local sections of $\mathcal{F}$. The flag of subsheaves

$$
\mathcal{F}=\mathcal{F}^{1} \subset \mathcal{F}^{2} \subset \cdots \subset T \mathcal{X}
$$

may be defined in the same manner as for distributions: set $\mathcal{F}^{1}=\mathcal{F}$, and

$$
\mathcal{F}^{i+1}=\mathcal{F}^{i}+\left[\mathcal{F}, \mathcal{F}^{i}\right], \quad i \geq 1 .
$$

At each point $x \in \mathcal{X}$, this gives a flag of affine subspaces of $T_{x} X$ :

$$
\mathcal{F}_{x} \subset \mathcal{F}_{x}^{2} \subset \cdots \subset T_{x} X
$$

Each affine distribution $\mathcal{F}^{i}$ in the flag has an associated direction distribution $L_{\mathcal{F}^{i}}$; these distributions clearly have the property that

$$
L_{\mathcal{F}}^{i} \subset L_{\mathcal{F} i} .
$$

The step, the growth vector, and the notion of bracket-generating are defined for affine distributions in the same way as for linear distributions. But we will also want to consider affine distributions with a slightly weaker bracket-generating property.

Definition 1.5. An affine distribution $\mathcal{F}$ on an $n$-dimensional manifold $X$ is almost bracketgenerating if $\operatorname{rank}\left(\mathcal{F}^{\infty}\right)=n-1$, and for each $x \in \mathcal{X}$ and any $\xi(x) \in \mathcal{F}_{x}, \operatorname{span}\left(\xi(x),\left(L_{\mathcal{F} \infty}\right)_{x}\right)=$ $T_{x} X$.

We also impose an additional condition in the definition of constant type - namely, that $\mathcal{F}$ is strictly affine, and that each element $\mathcal{F}^{i}$ is either strictly affine or a linear distribution. This condition is reflected in the second condition of the following definition.

Definition 1.6. The affine distribution $\mathcal{F}$ has constant type if

- $n_{i}(x)$ is constant on $\mathcal{X}$ for all $i$; i.e., the growth vector of $\mathcal{F}$ is constant on $\mathcal{X}$, and

- for any section $\xi$ of $\mathcal{F}, \operatorname{dim}\left(\operatorname{span}\left(\xi(x),\left(L_{\mathcal{F}^{i}}\right)_{x}\right)\right)$ is constant on $\mathcal{X}$ for all $i$. 
The well-known theorems of Frobenius, Pfaff, and Engel (cf. Section 2.1) imply that linear distributions of constant type on manifolds of dimension $n \leq 4$ have only integer-valued invariants: specifically, any two linear distributions of constant type on manifolds of the same dimension, with the same rank and growth vector, are locally equivalent via a diffeomorphism of the underlying manifolds. Beginning with $n=5$ and $s=2$, local invariants depending on arbitrary functions appear: the famous paper of É. Cartan [7] describes local invariants of rank 2 distributions on 5 -manifolds with growth vector $(2,3,5)$. More recently, Bryant [5] has described local invariants of rank 3 distributions on 6 -manifolds with growth vector $(3,6)$, and Doubrov and Zeleneko [10, 11] have given a fairly comprehensive treatment of maximally nonholonomic distributions of ranks 2 and 3 on manifolds of arbitrary dimension.

The study of affine distributions is more recent and less extensive. Elkin [12, 13] has studied equivalence of affine distributions on manifolds of dimension $n \leq 4$ under diffeomorphisms of the underlying manifolds, resulting in local normal forms for the associated control systems. Local invariants appear in lower dimensions than for linear distributions: the first local invariants arise in the case of rank 1 affine distributions on 3-dimensional manifolds. Local normal forms for generic rank 1 affine distributions on $\mathbb{R}^{3}$ have also been obtained by Agrachev [1] and Wilkens [25], and for generic rank 1 affine distributions on $\mathbb{R}^{n}$ and generic rank 2 affine distributions on $\mathbb{R}^{4}$ and $\mathbb{R}^{5}$ by Agrachev and Zelenko [3].

Given a control-affine system (1.3), one can canonically associate to it an affine distribution $\mathcal{F}$ on the state space $X$ : if we let $a_{1}(x), \ldots, a_{s}(x)$ denote the columns of the matrix $A(x)$, then

$$
\mathcal{F}_{x}=\left\{a_{0}(x)+\sum_{i=1}^{s} u^{i} a_{i}(x) \mid u^{1}, \ldots, u^{s} \in \mathbb{R}\right\} .
$$

Elkin defines two systems

$$
\begin{aligned}
& \dot{x}=a_{0}(x)+\sum_{i=1}^{s} u^{i} a_{i}(x), \\
& \dot{y}=b_{0}(y)+\sum_{i=1}^{s} v^{i} b_{i}(y)
\end{aligned}
$$

on state spaces $\mathcal{X}, \mathcal{Y}$, respectively, to be affine equivalent if there exists a diffeomorphism $\psi$ : $x \rightarrow y$ such that any absolutely continuous curve $x(t)$ in $X$ is a solution of (1.5) if and only if the curve $y(t)=\psi(x(t))$ is a solution of (1.6). More specifically, such a diffeomorphism must have the property that

$$
\begin{aligned}
& \psi_{*}\left(a_{0}(x)\right)=b_{0}(\psi(x))+\sum_{j=1}^{s} \lambda_{0}^{j}(x) b_{j}(\psi(x)), \\
& \psi_{*}\left(a_{i}(x)\right)=\sum_{j=1}^{s} \lambda_{i}^{j}(x) b_{j}(\psi(x)), \quad 1 \leq i \leq s
\end{aligned}
$$

for some functions $\lambda_{0}^{j}, \lambda_{i}^{j}$ on $\mathcal{X}$. In terms of the associated affine distributions $\mathcal{F}_{x}, \mathcal{F}_{y}$, this is equivalent to the statement that $\psi_{*}\left(\mathcal{F}_{X}\right)=\mathcal{F}_{y}$.

This notion of equivalence is quite natural, especially in terms of the geometry of affine distributions. But with an eye towards studying optimal control problems of the form (1.3), we propose a slightly more restrictive definition of equivalence. According to (1.7), the drift vector field $a_{0}(x)$ in (1.3) may be replaced by any vector field of the form $a_{0}(x)+\sum_{j=1}^{s} \lambda_{0}^{j}(x) a_{j}(x)$, and the resulting control system will be affine equivalent to the original. But in practice, there is often a preferred choice for the drift vector field, corresponding to a zero value for some physical 
control inputs. This is particularly true in optimal control, where there is typically a specific control input whose cost function is minimal. In this paper, therefore, we will study the following type of geometric object, corresponding to a control-affine system (1.3) with a fixed drift vector field $a_{0}$ :

Definition 1.7. A point-affine distribution on a manifold $\mathcal{X}$ is an affine distribution $\mathcal{F}$ on $X$, together with a distinguished vector field $a_{0} \in \mathcal{F}$.

We will consider the equivalence problem for point-affine distributions and their associated control-affine systems with respect to the following notion of equivalence:

Definition 1.8. The point-affine distributions

$$
\mathcal{F}_{\mathcal{X}}=a_{0}+\operatorname{span}\left(a_{1}, \ldots, a_{s}\right), \quad \mathcal{F}_{y}=b_{0}+\operatorname{span}\left(b_{1}, \ldots, b_{s}\right)
$$

on the manifolds $X, y$ (corresponding to the control-affine systems (1.5) and (1.6), respectively) will be called point-affine equivalent if there exists a diffeomorphism $\psi: X \rightarrow \mathcal{Y}$ such that

$$
\begin{aligned}
& \psi_{*}\left(a_{0}(x)\right)=b_{0}(\psi(x)), \\
& \psi_{*}\left(a_{i}(x)\right)=\sum_{j=1}^{s} \lambda_{i}^{j}(x) b_{j}(\psi(x)), \quad 1 \leq i \leq s
\end{aligned}
$$

for some functions $\lambda_{i}^{j}$ on $X$.

In other words, there must exist a diffeomorphism that preserves both the affine distributions and the distinguished vector fields.

The primary goal of this paper is the local classification of point-affine distributions of constant type with respect to point-affine equivalence. We will use Cartan's method of equivalence to compute local invariants for point-affine distributions. The remainder of the paper is organized as follows: in Section 2, we review some well-known results on the geometry of linear distributions of constant type, as well as some of Elkin's results on affine distributions, in order to put our results in context. In Section 3 we compute local invariants for point-affine distributions of constant type when $\operatorname{dim}(\mathcal{X})=n, \operatorname{rank}(\mathcal{F})=n-1$, and when $\operatorname{dim}(\mathcal{X})=3, \operatorname{rank}(\mathcal{F})=1$. In Section 4 we discuss examples and potential directions for further study.

\section{Review of prior results}

\subsection{Classical normal form results for linear distributions}

The following results are well-known; see, e.g., [4] or [16]. We include them here primarily to provide historical context for our results; some of them will also be used in the proofs in Section 3 .

Definition 2.1. A distribution $\mathcal{D}$ on a manifold $X$ is called completely integrable (or, more succinctly, integrable), if $\mathcal{D}^{\infty}=\mathcal{D}$.

Theorem 2.2 (Frobenius). Let $\mathcal{D}$ be a rank $s$ distribution on an $n$-dimensional manifold $X$. If $\mathcal{D}$ is completely integrable, then in a sufficiently small neighborhood of any point $x \in \mathcal{X}$, there exist local coordinates $\left(x^{1}, \ldots, x^{n}\right)$ such that

$$
\mathcal{D}=\operatorname{span}\left(\frac{\partial}{\partial x^{1}}, \ldots, \frac{\partial}{\partial x^{s}}\right)
$$

or, equivalently,

$$
\mathcal{D}^{\perp}=\left\{d x^{s+1}, \ldots, d x^{n}\right\} .
$$


Theorem 2.3 (Pfaff). Let $\mathcal{D}$ be a rank $n$ distribution on an $(n+1)$-dimensional manifold $\mathcal{X}$, and let $\theta$ be a nonvanishing 1 -form on $X$ such that $\mathcal{D}=\{\theta\}^{\perp}$. Let $k$ be the integer defined by the conditions

$$
\theta \wedge(d \theta)^{k} \neq 0, \quad \theta \wedge(d \theta)^{k+1}=0 .
$$

( $k$ is called the Pfaff rank of $\theta$.) In a sufficiently small neighborhood of any point $x \in \mathcal{X}$ on which $k$ is constant, there exist local coordinates $\left(x^{0}, \ldots, x^{n}\right)$ such that

$$
\theta= \begin{cases}d x^{1}+x^{2} d x^{3}+\cdots+x^{2 k} d x^{2 k+1} & \text { if }(d \theta)^{k+1}=0 \\ x^{0} d x^{1}+x^{2} d x^{3}+\cdots+x^{2 k} d x^{2 k+1} & \text { if }(d \theta)^{k+1} \neq 0 .\end{cases}
$$

Theorem 2.4 (Engel). Let $\mathcal{D}$ be a rank 2 distribution of constant type on a 4-dimensional manifold $X$, with growth vector $(2,3,4)$. Then in a sufficiently small neighborhood of any point $x \in \mathcal{X}$, there exist local coordinates $\left(x^{1}, x^{2}, x^{3}, x^{4}\right)$ such that

$$
\mathcal{D}^{\perp}=\left\{d x^{2}-x^{3} d x^{1}, d x^{3}-x^{4} d x^{1}\right\} .
$$

\subsection{Local classification of rank 1 affine systems on 2- and 3-dimensional manifolds under affine equivalence}

Linear distributions of rank 1 have no local invariants, as all nonvanishing vector fields on a manifold are well-known to be locally equivalent. Affine distributions, however, may have local invariants even in rank 1 . The following results are due to Elkin $[12,13]$.

Theorem 2.5 (Elkin). Let $\mathcal{F}$ be a rank 1 strictly affine distribution of constant type on a 2dimensional manifold $X$.

1. If $\mathcal{F}$ is almost bracket-generating, then in a sufficiently small neighborhood of any point $x \in X$, there exist local coordinates $\left(x^{1}, x^{2}\right)$ such that

$$
\mathcal{F}=\frac{\partial}{\partial x^{1}}+\operatorname{span}\left(\frac{\partial}{\partial x^{2}}\right)
$$

2. If $\mathcal{F}$ is bracket-generating, then in a sufficiently small neighborhood of any point $x \in X$, there exist local coordinates $\left(x^{1}, x^{2}\right)$ such that

$$
\mathcal{F}=x^{2} \frac{\partial}{\partial x^{1}}+\operatorname{span}\left(\frac{\partial}{\partial x^{2}}\right) .
$$

Note that, for reasons of dimension, any strictly affine distribution of rank 1 on a 2-manifold is either almost bracket-generating or bracket-generating.

Theorem 2.6 (Elkin). Let $\mathcal{F}$ be a rank 1 strictly affine distribution of constant type on a 3dimensional manifold $X$.

1. If $\mathcal{F}$ is almost bracket-generating, then in a sufficiently small neighborhood of any point $x \in \mathcal{X}$, there exist local coordinates $\left(x^{1}, x^{2}, x^{3}\right)$ such that

$$
\mathcal{F}=\left(\frac{\partial}{\partial x^{1}}+x^{3} \frac{\partial}{\partial x^{2}}\right)+\operatorname{span}\left(\frac{\partial}{\partial x^{3}}\right) .
$$


2. If $\mathcal{F}$ is bracket-generating and $L_{\mathcal{F}^{2}}$ (which must have rank 2$)$ is Frobenius, then in a sufficiently small neighborhood of any point $x \in \mathcal{X}$, there exist local coordinates $\left(x^{1}, x^{2}, x^{3}\right)$ such that

$$
\mathcal{F}=\left(x^{2} \frac{\partial}{\partial x^{1}}+x^{3} \frac{\partial}{\partial x^{2}}\right)+\operatorname{span}\left(\frac{\partial}{\partial x^{3}}\right) .
$$

3. If $\mathcal{F}$ is bracket-generating and $L_{\mathcal{F}^{2}}$ is not Frobenius, then in a sufficiently small neighborhood of any point $x \in \mathcal{X}$, there exist local coordinates $\left(x^{1}, x^{2}, x^{3}\right)$ such that

$$
\mathcal{F}=\frac{\partial}{\partial x^{1}}+\operatorname{span}\left(x^{3} \frac{\partial}{\partial x^{1}}+\frac{\partial}{\partial x^{2}}+H \frac{\partial}{\partial x^{3}}\right),
$$

where $H$ is an arbitrary function on $X$ satisfying $\frac{\partial H}{\partial x^{1}} \neq 0$.

Note that if a rank 1 strictly affine distribution $\mathcal{F}$ on a 3 -manifold is not bracket-generating or almost bracket-generating, then $\operatorname{span}(\mathcal{F})$ is a rank 2 Frobenius distribution $\mathcal{D}_{\mathcal{F}}$ on $\mathcal{X}$, and $\mathcal{F}$ may be regarded (at least locally) as living on the 2-dimensional leaves of the foliation which is tangent to $\mathcal{D}_{\mathcal{F}}$.

\section{Local point-affine equivalence results for point-affine distributions}

\subsection{Equivalence problem for rank 1 point-affine distributions on 2-manifolds}

For point-affine equivalence, the first local invariants appear in the simplest nontrivial case: rank 1 point-affine distributions on 2-dimensional manifolds.

Theorem 3.1. Let $\mathcal{F}$ be a rank 1 strictly affine point-affine distribution of constant type on a 2-dimensional manifold $X$.

1. If $\mathcal{F}$ is almost bracket-generating, then in a sufficiently small neighborhood of any point $x \in \mathcal{X}$, there exist local coordinates $\left(x^{1}, x^{2}\right)$ such that

$$
\mathcal{F}=\frac{\partial}{\partial x^{1}}+\operatorname{span}\left(\frac{\partial}{\partial x^{2}}\right)
$$

2. If $\mathcal{F}$ is bracket-generating, then in a sufficiently small neighborhood of any point $x \in \mathcal{X}$, there exist local coordinates $\left(x^{1}, x^{2}\right)$ such that

$$
\mathcal{F}=x^{2}\left(\frac{\partial}{\partial x^{1}}+J \frac{\partial}{\partial x^{2}}\right)+\operatorname{span}\left(\frac{\partial}{\partial x^{2}}\right)
$$

where $J$ is an arbitrary function on $X$.

Proof. We will employ Cartan's method of equivalence in this proof. Let $\mathcal{F}$ be a rank 1 pointaffine distribution of constant type on a 2-dimensional manifold $\mathcal{X}$. A local framing - i.e., a pair of everywhere linearly independent vector fields $\left(v_{1}, v_{2}\right)$ on $\mathcal{X}$ - will be called admissible if $v_{1}$ is the distinguished vector field and

$$
\mathcal{F}=v_{1}+\operatorname{span}\left(v_{2}\right) .
$$


If $\left(v_{1}, v_{2}\right)$ is any admissible framing on $\mathcal{X}$, then any other admissible framing $\left(\tilde{v}_{1}, \tilde{v}_{2}\right)$ has the form

$$
\tilde{v}_{1}=v_{1}, \quad \tilde{v}_{2}=b_{2} v_{2}
$$

for some nonvanishing function $b_{2}$ on $X$.

A local coframing $\left(\bar{\eta}^{1}, \bar{\eta}^{2}\right)$ on $\mathcal{X}$ will be called 0 -adapted if it is the dual coframing of some admissible framing. Any two 0 -adapted coframings on $\mathcal{X}$ vary by a transformation of the form

$$
\left[\begin{array}{c}
\tilde{\bar{\eta}}^{1} \\
\tilde{\bar{\eta}}^{2}
\end{array}\right]=\left[\begin{array}{cc}
1 & 0 \\
0 & b_{2}
\end{array}\right]^{-1}\left[\begin{array}{c}
\bar{\eta}^{1} \\
\bar{\eta}^{2}
\end{array}\right],
$$

with $b_{2} \neq 0$.

The 0 -adapted coframings are the local sections of a principal fiber bundle $\pi: \mathcal{B}_{0} \rightarrow \mathcal{X}$, with structure group $G_{0} \subset G L(2)$ consisting of all invertible matrices of the form in (3.1). The right action of $G_{0}$ on sections $\sigma: \mathcal{X} \rightarrow \mathcal{B}_{0}$ is given by $\sigma \cdot g=g^{-1} \sigma$. (This is the reason for the inverse occurring in (3.1).)

There exist canonical 1-forms $\eta^{1}, \eta^{2}$ on $\mathcal{B}_{0}$ with the reproducing property that for any coframing $\left(\bar{\eta}^{1}, \bar{\eta}^{2}\right)$ given by a local section $\sigma: \mathcal{X} \rightarrow \mathcal{B}_{0}$,

$$
\sigma^{*}\left(\eta^{i}\right)=\bar{\eta}^{i}
$$

These are referred to as the semi-basic forms on $\mathcal{B}_{0}$. A standard argument shows that there also exists a (non-unique) 1-form $\beta_{2}$ (referred to as a pseudo-connection form or, more succinctly, a connection form), linearly independent from the semi-basic forms, and a function $T_{12}^{1}$ on $\mathcal{B}_{0}$ (referred to as a torsion function) such that

$$
\left[\begin{array}{l}
d \eta^{1} \\
d \eta^{2}
\end{array}\right]=-\left[\begin{array}{ll}
0 & 0 \\
0 & \beta_{2}
\end{array}\right]\left[\begin{array}{l}
\eta^{1} \\
\eta^{2}
\end{array}\right]+\left[\begin{array}{c}
T_{12}^{1} \eta^{1} \wedge \eta^{2} \\
0
\end{array}\right] .
$$

(See [15] for details about the method of equivalence.) These are the structure equations of the $G_{0}$-structure $\mathcal{B}_{0}$. The semi-basic forms and the connection form together form a local coframing on $\mathcal{B}_{0}$.

We proceed with the method of equivalence by examining how the function $T_{12}^{1}$ varies if we change from one 0 -adapted coframing to another. A straightforward computation shows that under a transformation of the form (3.1), we have

$$
\tilde{T}_{12}^{1}=b_{2} T_{12}^{1} \text {. }
$$

Thus $T_{12}^{1}$ is a relative invariant: if it vanishes for any coframing at a point $x \in \mathcal{X}$, then it vanishes for every coframing at $x$.

At this point, we need to divide into cases based on whether or not $T_{12}^{1}$ vanishes.

Case 1. Suppose that $T_{12}^{1}=0$. Then the structure equations (3.2) contain no local invariants, so no further adaptations can be made. The tableau consisting of all matrices of the form in (3.2) is involutive with Cartan character $s_{1}=1$.

Given any 0 -adapted coframing, consider the dual framing $\left(v_{1}, v_{2}\right)$. The structure equations imply that

$$
\left[v_{1}, v_{2}\right] \equiv 0 \quad \bmod v_{2}
$$

therefore, $\mathcal{F}$ is almost bracket-generating. In order to recover the normal form of Theorem 3.1, let $\left(\bar{\eta}^{1}, \bar{\eta}^{2}\right)$ be any 0 -adapted local coframing on $\mathcal{X}$ given by a local section $\sigma: \mathcal{X} \rightarrow \mathcal{B}_{0}$, and consider the pullbacks of the structure equations (3.2) via $\sigma$ :

$$
d \bar{\eta}^{1}=0, \quad d \bar{\eta}^{2}=-\bar{\beta}_{2} \wedge \bar{\eta}^{2} .
$$


(Note that the pullback $\bar{\beta}_{2}$ of the connection form $\beta_{2}$ is a 1 -form on $X$, so it is no longer linearly independent from $\bar{\eta}^{1}, \bar{\eta}^{2}$.)

Since $\bar{\eta}^{1}$ is an exact 1 -form, we can choose a local coordinate $x^{1}$ on $\mathcal{X}$ such that $\bar{\eta}^{1}=d x^{1}$. Then, since $\bar{\eta}^{2}$ is an integrable 1 -form, we can choose a local coordinate $x^{2}$ on $\mathcal{X}$, functionally independent from $x^{1}$, such that

$$
\bar{\eta}^{2}=\lambda d x^{2}
$$

for some nonvanishing function $\lambda$ on $X$. By replacing the given coframing with the (also 0adapted) coframing $\left(\bar{\eta}^{1}, \lambda^{-1} \bar{\eta}^{2}\right)$, we can assume that $\lambda \equiv 1$. Our 1-adapted coframing now has the form

$$
\bar{\eta}^{1}=d x^{1}, \quad \bar{\eta}^{2}=d x^{2} .
$$

The dual framing is

$$
v_{1}=\frac{\partial}{\partial x^{1}}, \quad v_{2}=\frac{\partial}{\partial x^{2}},
$$

and

$$
\mathcal{F}=\frac{\partial}{\partial x^{1}}+\operatorname{span}\left(\frac{\partial}{\partial x^{2}}\right)
$$

Case 2. Suppose that $T_{12}^{1} \neq 0$. (3.3) implies that, given any 0 -adapted coframing $\left(\bar{\eta}^{1}, \bar{\eta}^{2}\right)$, we can perform a transformation of the form (3.1) to arrive at a new 0-adapted coframing for which $T_{12}^{1}=1$. Such a coframing will be called 1-adapted.

Any two 1 -adapted coframings on $X$ vary by a transformation of the form

$$
\left[\begin{array}{c}
\tilde{\bar{\eta}}^{1} \\
\tilde{\bar{\eta}}^{2}
\end{array}\right]=\left[\begin{array}{ll}
1 & 0 \\
0 & 1
\end{array}\right]^{-1}\left[\begin{array}{l}
\bar{\eta}^{1} \\
\bar{\eta}^{2}
\end{array}\right] .
$$

In other words, the 1-adapted coframings are the local sections of an (e)-structure $\mathcal{B}_{1} \subset \mathcal{B}_{0}$. The projection $\pi: \mathcal{B}_{1} \rightarrow \mathcal{X}$ is a diffeomorphism, and there is a unique 1-adapted coframing $\left(\bar{\eta}^{1}, \bar{\eta}^{2}\right)$ on $X$. This coframing has structure equations

$$
\begin{aligned}
& d \bar{\eta}^{1}=\bar{\eta}^{1} \wedge \bar{\eta}^{2}, \\
& d \bar{\eta}^{2}=T_{12}^{2} \bar{\eta}^{1} \wedge \bar{\eta}^{2},
\end{aligned}
$$

for some function $T_{12}^{2}$ on $\mathcal{X}$.

Consider the dual framing $\left(v_{1}, v_{2}\right)$ of a 1 -adapted coframing. The structure equations $(3.4)$ imply that

$$
\left[v_{1}, v_{2}\right] \equiv-v_{1} \quad \bmod v_{2}
$$

therefore, $\mathcal{F}$ is bracket-generating. Since $\bar{\eta}^{1}$ is integrable but not exact, we can choose local coordinates $x^{1}, x^{2}$ on $X$ such that $\bar{\eta}^{1}=\frac{1}{x^{2}} d x^{1}$. Then the first equation in (3.4) implies that

$$
\bar{\eta}^{2}=\frac{1}{x^{2}}\left(d x^{2}-J d x^{1}\right)
$$

for some function $J$ on $X$. The second equation then implies that

$$
T_{12}^{2}=x^{2} J_{x^{2}}-J .
$$


In terms of these local coordinates, the dual framing is

$$
v_{1}=x^{2}\left(\frac{\partial}{\partial x^{1}}+J \frac{\partial}{\partial x^{2}}\right), \quad v_{2}=x^{2} \frac{\partial}{\partial x^{2}}
$$

and $\operatorname{since} \operatorname{span}\left(v_{2}\right)=\operatorname{span}\left(\frac{\partial}{\partial x^{2}}\right)$

$$
\mathcal{F}=x^{2}\left(\frac{\partial}{\partial x^{1}}+J \frac{\partial}{\partial x^{2}}\right)+\operatorname{span}\left(\frac{\partial}{\partial x^{2}}\right) .
$$

Remark 3.2. This choice of coordinates $x^{1}, x^{2}$ such that $\bar{\eta}^{1}=\frac{1}{x^{2}} d x^{1}$ is unique up to coordinate transformations of the form

$$
x^{1} \rightarrow f\left(\tilde{x}^{1}\right), \quad x^{2} \rightarrow f^{\prime}\left(\tilde{x}^{1}\right) \tilde{x}^{2} .
$$

Under a transformation of the form (3.6), we have

$$
J\left(x^{1}, x^{2}\right) \rightarrow J\left(f\left(\tilde{x}^{1}\right), f^{\prime}\left(\tilde{x}^{1}\right) \tilde{x}^{2}\right)-\frac{f^{\prime \prime}\left(\tilde{x}^{1}\right)}{f^{\prime}\left(\tilde{x}^{1}\right)} \tilde{x}^{2} .
$$

Therefore, an admissible change of coordinates can only change $J$ by a linear function of $x^{2}$, and point-affine distributions of this type locally depend essentially on one arbitrary function of 2 variables. In particular, the point-affine distribution (3.5) is locally equivalent to the "flat" case $J=0$ if and only if $J=g\left(x^{1}\right) x^{2}$ for some function $g\left(x^{1}\right)$.

\subsection{Equivalence problem for rank $(n-1)$ point-affine distributions on $n$-manifolds}

Theorem 3.1 is a special case of the following more general theorem:

Theorem 3.3. Let $\mathcal{F}$ be a rank $(n-1)$ strictly affine point-affine distribution of constant type on an $n$-dimensional manifold $X$. Let $L_{\mathcal{F}}$ be the associated direction distribution of rank $(n-1)$, let $v_{1}$ denote the distinguished vector field, and let $\bar{\eta}^{1}$ be the unique 1-form on $X$ satisfying

$$
L_{\mathcal{F}}=\left(\bar{\eta}^{1}\right)^{\perp}, \quad \bar{\eta}^{1}\left(v_{1}\right)=1 .
$$

Let $k$ denote the Pfaff rank of $\bar{\eta}^{1}$, i.e., the unique integer such that

$$
\bar{\eta}^{1} \wedge\left(d \bar{\eta}^{1}\right)^{k} \neq 0, \quad \bar{\eta}^{1} \wedge\left(d \bar{\eta}^{1}\right)^{k+1}=0 .
$$

1. If $\left(d \bar{\eta}^{1}\right)^{k+1}=0$, then in a sufficiently small neighborhood of any point $x \in X$ on which $k$ is constant, there exist local coordinates $\left(x^{1}, \ldots, x^{n}\right)$ such that

$$
\begin{aligned}
\mathcal{F}= & \left(1+\sum_{r=1}^{k} x^{k+r+1} J_{k+r+1}\right) \frac{\partial}{\partial x^{1}}+\sum_{r=1}^{k}\left(J_{k+r+1} \frac{\partial}{\partial x^{r+1}}-J_{r+1} \frac{\partial}{\partial x^{k+r+1}}\right) \\
& +\operatorname{span}\left(\left(\frac{\partial}{\partial x^{2}}+x^{k+2} \frac{\partial}{\partial x^{1}}\right), \ldots,\left(\frac{\partial}{\partial x^{k+1}}+x^{2 k+1} \frac{\partial}{\partial x^{1}}\right), \frac{\partial}{\partial x^{k+2}}, \ldots, \frac{\partial}{\partial x^{n}}\right),
\end{aligned}
$$

where $J_{2}, \ldots, J_{2 k+1}$ are arbitrary functions on $\mathcal{X}$. 
2. If $\left(d \bar{\eta}^{1}\right)^{k+1} \neq 0$, then in a sufficiently small neighborhood of any point $x \in \mathcal{X}$ on which $k$ is constant, there exist local coordinates $\left(x^{1}, \ldots, x^{n}\right)$ such that

$$
\begin{aligned}
\mathcal{F}=\left(x^{2 k+2}+\right. & \left.\sum_{r=1}^{k} x^{k+r+1} J_{k+r+1}\right) \frac{\partial}{\partial x^{1}}+\sum_{r=1}^{k}\left(J_{k+r+1} \frac{\partial}{\partial x^{r+1}}-J_{r+1} \frac{\partial}{\partial x^{k+r+1}}\right)-J_{1} \frac{\partial}{\partial x^{2 k+2}} \\
+\operatorname{span}( & \left(x^{2 k+2}\left(\frac{\partial}{\partial x^{2}}+x^{k+2} \frac{\partial}{\partial x^{1}}\right)-J_{2} \frac{\partial}{\partial x^{2 k+2}}\right), \ldots, \\
& \left(x^{2 k+2}\left(\frac{\partial}{\partial x^{k+1}}+x^{2 k+1} \frac{\partial}{\partial x^{1}}\right)-J_{k+1} \frac{\partial}{\partial x^{2 k+2}}\right), \\
& \left(\frac{\partial}{\partial x^{k+2}}-J_{k+2} \frac{\partial}{\partial x^{2 k+2}}\right), \ldots,\left(\frac{\partial}{\partial x^{2 k+1}}-J_{2 k+1} \frac{\partial}{\partial x^{2 k+2}}\right), \\
& \left.\frac{\partial}{\partial x^{2 k+2}}, \ldots, \frac{\partial}{\partial x^{n}}\right)
\end{aligned}
$$

where $J_{1}, \ldots, J_{2 k+1}$ are arbitrary functions on $\mathcal{X}$.

Proof. Let $\mathcal{F}$ be a rank $(n-1)$ point-affine distribution of constant type on an $n$-dimensional manifold $\mathcal{X}$. A local framing $\left(v_{1}, \ldots, v_{n}\right)$ on $\mathcal{X}$ will be called admissible if $v_{1}$ is the distinguished vector field and

$$
\mathcal{F}=v_{1}+\operatorname{span}\left(v_{2}, \ldots, v_{n}\right) .
$$

A local coframing $\left(\bar{\eta}^{1}, \ldots, \bar{\eta}^{n}\right)$ on $\mathcal{X}$ will be called admissible if, for any admissible framing $\left(v_{1}, \ldots, v_{n}\right)$,

$$
\begin{array}{ll}
\bar{\eta}^{1}\left(v_{1}\right)=1, & \\
\bar{\eta}^{1}\left(v_{j}\right)=0, & 2 \leq j \leq n, \\
\bar{\eta}^{j}\left(v_{1}\right)=0, & 2 \leq j \leq n .
\end{array}
$$

Any two admissible coframings on $X$ vary by a transformation of the form

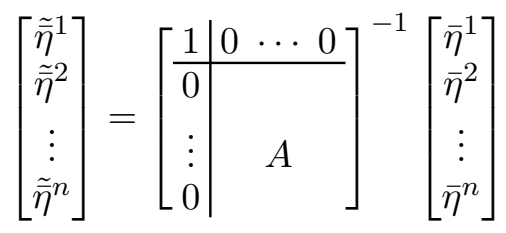

for some matrix $A \in G L(n-1, \mathbb{R})$.

An admissible coframing will be called 0-adapted if

$$
d \bar{\eta}^{1} \equiv \sum_{r=1}^{k} \bar{\eta}^{r+1} \wedge \bar{\eta}^{k+r+1} \quad \bmod \bar{\eta}^{1},
$$

where $k$ is the Pfaff rank of $\bar{\eta}^{1}$. Since the Cartan system

$$
\mathcal{C}\left(\bar{\eta}^{1}\right)=\left\{\bar{\eta}^{1}, \ldots, \bar{\eta}^{2 k+1}\right\}
$$

and the system

$$
v_{1}^{\perp}=\left\{\bar{\eta}^{2}, \ldots, \bar{\eta}^{n}\right\}
$$

are both well-defined independent of the choice of 0-adapted coframing, the subsystem

$$
\mathcal{C}\left(\bar{\eta}^{1}\right) \cap v_{1}^{\perp}=\left\{\bar{\eta}^{2}, \ldots, \bar{\eta}^{2 k+1}\right\}
$$


is also well-defined. Thus any two 0 -adapted coframings on $\mathcal{X}$ vary by a transformation of the form

$$
\left[\begin{array}{c}
\tilde{\bar{\eta}}^{1} \\
\tilde{\bar{\eta}}^{2} \\
\vdots \\
\tilde{\bar{\eta}}^{2 k+1} \\
\tilde{\bar{\eta}}^{2 k+2} \\
\vdots \\
\tilde{\bar{\eta}}^{n}
\end{array}\right]\left[\begin{array}{c|ccc|ccc}
1 & 0 & \cdots & 0 & 0 & \cdots & 0 \\
\hline 0 & & & 0 & \cdots & 0 \\
\vdots & & A & \vdots & & \vdots \\
0 & & & 0 & \cdots & 0 \\
\hline 0 & & & & \\
\vdots & & B & & C &
\end{array}\right]^{-1}\left[\begin{array}{c}
\bar{\eta}^{1} \\
\bar{\eta}^{2} \\
\vdots \\
\bar{\eta}^{2 k+1} \\
\bar{\eta}^{2 k+2} \\
\vdots \\
\bar{\eta}^{n}
\end{array}\right]
$$

where $A \in S p(2 k, \mathbb{R}), B \in M_{(n-2 k-1) \times(2 k)}(\mathbb{R}), C \in G L(n-2 k-1, \mathbb{R})$.

The bundle $\pi: \mathcal{B}_{0} \rightarrow \mathcal{X}$ of 0 -adapted coframings with structure group $G_{0}$ consisting of matrices of the form (3.7) is defined as in Section 3.1, as are the semi-basic forms $\eta^{1}, \ldots, \eta^{n}$ on $\mathcal{B}_{0}$. The first structure equation of $\mathcal{B}_{0}$ is

$$
d \eta^{1}=\eta^{1} \wedge \phi+\sum_{r=1}^{k} \eta^{r+1} \wedge \eta^{k+r+1},
$$

where $\phi \in \pi^{*}\left(v_{1}^{\perp}\right)$. There are two cases to consider:

1. $\phi \in \mathcal{C}\left(\eta^{1}\right)$;

2. $\phi \notin \mathcal{C}\left(\eta^{1}\right)$.

Case 1: $\phi \in \mathcal{C}\left(\eta^{1}\right)$. Then

$$
\left(d \eta^{1}\right)^{k+1}=0 .
$$

Let $\sigma: X \rightarrow \mathcal{B}_{0}$ be any local section of $\mathcal{B}_{0}$ (i.e., a 0 -adapted coframing $\left(\bar{\eta}^{1}, \ldots, \bar{\eta}^{n}\right)$ on $\mathcal{X}$ ). By the reproducing property of the semi-basic forms,

$$
\left(d \bar{\eta}^{1}\right)^{k+1}=0,
$$

so by the Pfaff theorem there exist local coordinates $x^{1}, \ldots, x^{2 k+1}$ on $X$ such that

$$
\bar{\eta}^{1}=d x^{1}-\sum_{r=1}^{k} x^{k+r+1} d x^{r+1} .
$$

We can write

$$
\bar{\phi}=\sum_{j=1}^{2 k} J_{j+1} \bar{\eta}^{j+1}
$$

for some functions $J_{2}, \ldots, J_{2 k+1}$ on $X$. Then we have

$$
\begin{aligned}
d \bar{\eta}^{1} & =\bar{\eta}^{1} \wedge\left(\sum_{j=1}^{2 k} J_{j+1} \bar{\eta}^{j+1}\right)+\sum_{r=1}^{k} \bar{\eta}^{r+1} \wedge \bar{\eta}^{k+r+1} \\
& =\sum_{r=1}^{k}\left(\bar{\eta}^{r+1}+J_{k+r+1} \bar{\eta}^{1}\right) \wedge\left(\bar{\eta}^{k+r+1}-J_{r+1} \bar{\eta}^{1}\right)=\sum_{r=1}^{k} d x^{r+1} \wedge d x^{k+r+1} .
\end{aligned}
$$


Thus we can choose a 0 -adapted coframing with

$$
\left.\begin{array}{l}
\bar{\eta}^{s+1}=d x^{s+1}-J_{k+s+1} \bar{\eta}^{1} \\
\bar{\eta}^{k+s+1}=d x^{k+s+1}+J_{s+1} \bar{\eta}^{1}
\end{array}\right\}, \quad 1 \leq s \leq k .
$$

For reasons of dimension, the system

$$
v_{1}^{\perp}=\left\{\bar{\eta}^{2}, \ldots, \bar{\eta}^{n}\right\}
$$

is Frobenius; therefore, we can extend the local coordinates $x^{1}, \ldots, x^{2 k+1}$ to a full local coordinate system $x^{1}, \ldots, x^{n}$ with the property that

$$
v_{1}^{\perp}=\left\{\bar{\eta}^{2}, \ldots, \bar{\eta}^{2 k+1}, d x^{2 k+2}, \ldots, d x^{n}\right\}
$$

we can then choose the remainder of our 0-adapted coframing to be

$$
\bar{\eta}^{j}=d x^{j}, \quad 2 k+2 \leq j \leq n .
$$

The dual framing to the coframing defined by (3.8), (3.9), and (3.10) is:

$$
\begin{aligned}
& v_{1}=\left(1+\sum_{r=1}^{k} x^{k+r+1} J_{k+r+1}\right) \frac{\partial}{\partial x^{1}}+\sum_{r=1}^{k}\left(J_{k+r+1} \frac{\partial}{\partial x^{r+1}}-J_{r+1} \frac{\partial}{\partial x^{k+r+1}}\right), \\
& v_{s}=\frac{\partial}{\partial x^{s}}+x^{k+s} \frac{\partial}{\partial x^{1}}, \quad 2 \leq s \leq k+1, \\
& v_{j}=\frac{\partial}{\partial x^{j}}, \quad k+2 \leq j \leq n .
\end{aligned}
$$

The corresponding point-affine distribution $\mathcal{F}$ is:

$$
\begin{aligned}
\mathcal{F}= & \left(1+\sum_{r=1}^{k} x^{k+r+1} J_{k+r+1}\right) \frac{\partial}{\partial x^{1}}+\sum_{r=1}^{k}\left(J_{k+r+1} \frac{\partial}{\partial x^{r+1}}-J_{r+1} \frac{\partial}{\partial x^{k+r+1}}\right) \\
& +\operatorname{span}\left(\left(\frac{\partial}{\partial x^{2}}+x^{k+2} \frac{\partial}{\partial x^{1}}\right), \ldots,\left(\frac{\partial}{\partial x^{k+1}}+x^{2 k+1} \frac{\partial}{\partial x^{1}}\right), \frac{\partial}{\partial x^{k+2}}, \ldots, \frac{\partial}{\partial x^{n}}\right) .
\end{aligned}
$$

Note that our choices of local coordinates were arbitrary up to at most functions of $(n-1)$ variables, while the local invariants $J_{2}, \ldots, J_{2 k+1}$ are arbitrary functions of $n$ variables. Therefore, point-affine distributions of this type locally depend essentially on $2 k$ arbitrary functions of $n$ variables.

Case 2: $\phi \notin \mathcal{C}\left(\eta^{1}\right)$. Then

$$
\left(d \eta^{1}\right)^{k+1} \neq 0 .
$$

Let $\sigma: X \rightarrow \mathcal{B}_{0}$ be any local section of $\mathcal{B}_{0}$ (i.e., a 0 -adapted coframing $\left(\bar{\eta}^{1}, \ldots, \bar{\eta}^{n}\right)$ on $X$ ). Because $d \eta^{1}$ is semi-basic, it follows from the reproducing property of the semi-basic forms that

$$
\left(d \bar{\eta}^{1}\right)^{k+1} \neq 0 .
$$

By the Pfaff theorem, there exist local coordinates $x^{1}, \ldots, x^{2 k+2}$ on $X$ such that

$$
\bar{\eta}^{1}=\frac{1}{x^{2 k+2}}\left(d x^{1}-\sum_{r=1}^{k} x^{k+r+1} d x^{r+1}\right) .
$$


Then we have

$$
\begin{aligned}
d \bar{\eta}^{1} & =\bar{\eta}^{1} \wedge \bar{\phi}+\sum_{r=1}^{k} \bar{\eta}^{r+1} \wedge \bar{\eta}^{k+r+1} \\
& =\bar{\eta}^{1} \wedge\left(\frac{d x^{2 k+2}}{x^{2 k+2}}\right)+\frac{1}{x^{2 k+2}} \sum_{r=1}^{k} d x^{r+1} \wedge d x^{k+r+1}
\end{aligned}
$$

Therefore,

$$
\sum_{r=1}^{k} \bar{\eta}^{r+1} \wedge \bar{\eta}^{k+r+1} \equiv \frac{1}{x^{2 k+2}} \sum_{r=1}^{k} d x^{r+1} \wedge d x^{k+r+1} \quad \bmod \bar{\eta}^{1} .
$$

Thus we can choose a 0 -adapted coframing with

$$
\left.\begin{array}{l}
\bar{\eta}^{s+1}=\frac{1}{x^{2 k+2}}\left(d x^{s+1}-J_{k+s+1} \bar{\eta}^{1}\right) \\
\bar{\eta}^{k+s+1}=d x^{k+s+1}+J_{s+1} \bar{\eta}^{1}
\end{array}\right\}, \quad 1 \leq s \leq k,
$$

for some functions $J_{2}, \ldots, J_{2 k+1}$ on $\mathcal{X}$. Then we have

$$
\bar{\phi} \equiv \frac{1}{x^{2 k+2}}\left(d x^{2 k+2}+\sum_{j=1}^{2 k} J_{j+1} \bar{\eta}^{j+1}\right) \quad \bmod \bar{\eta}^{1} .
$$

The requirement that $\phi \in \pi^{*}\left(v_{1}^{\perp}\right)$ is equivalent to $\bar{\phi} \in v_{1}^{\perp}$, and this determines a unique function $J_{1}$ on $X$ such that

$$
\bar{\phi}=\frac{1}{x^{2 k+2}}\left(d x^{2 k+2}+\sum_{j=0}^{2 k} J_{j+1} \bar{\eta}^{j+1}\right) .
$$

We can now set

$$
\bar{\eta}^{2 k+2}=\bar{\phi}=\frac{1}{x^{2 k+2}}\left(d x^{2 k+2}+\sum_{j=0}^{2 k} J_{j+1} \bar{\eta}^{j+1}\right),
$$

and a similar argument to that given in the previous case shows that we can extend the local coordinates $x^{1}, \ldots, x^{2 k+2}$ to a full local coordinate system $x^{1}, \ldots, x^{n}$ and choose the remainder of our 0 -adapted coframing to be

$$
\bar{\eta}^{j}=d x^{j}, \quad 2 k+3 \leq j \leq n .
$$

The dual framing to the coframing defined by (3.11), (3.12), (3.13), and (3.14) is:

$$
\begin{aligned}
& v_{1}=\left(x^{2 k+2}+\sum_{r=1}^{k} x^{k+r+1} J_{k+r+1}\right) \frac{\partial}{\partial x^{1}}+\sum_{r=1}^{k}\left(J_{k+r+1} \frac{\partial}{\partial x^{r+1}}-J_{r+1} \frac{\partial}{\partial x^{k+r+1}}\right)-J_{1} \frac{\partial}{\partial x^{2 k+2}}, \\
& v_{s}=x^{2 k+2}\left(\frac{\partial}{\partial x^{s}}+x^{k+s} \frac{\partial}{\partial x^{1}}\right)-J_{s} \frac{\partial}{\partial x^{2 k+2}}, \quad 2 \leq s \leq k+1, \\
& v_{s}=\frac{\partial}{\partial x^{s}}-J_{s} \frac{\partial}{\partial x^{2 k+2}}, \quad k+2 \leq s \leq 2 k+1, \\
& v_{2 k+2}=x^{2 k+2} \frac{\partial}{\partial x^{2 k+2}}, \quad v_{j}=\frac{\partial}{\partial x^{j}}, \quad 2 k+3 \leq j \leq n .
\end{aligned}
$$


Since $\operatorname{span}\left(v_{2 k+2}\right)=\operatorname{span}\left(\frac{\partial}{\partial x^{2 k+2}}\right)$, the corresponding point-affine distribution $\mathcal{F}$ is:

$$
\begin{aligned}
\mathcal{F}=\left(x^{2 k+2}+\right. & \left.\sum_{r=1}^{k} x^{k+r+1} J_{k+r+1}\right) \frac{\partial}{\partial x^{1}}+\sum_{r=1}^{k}\left(J_{k+r+1} \frac{\partial}{\partial x^{r+1}}-J_{r+1} \frac{\partial}{\partial x^{k+r+1}}\right)-J_{1} \frac{\partial}{\partial x^{2 k+2}} \\
+\operatorname{span} & \left(\left(x^{2 k+2}\left(\frac{\partial}{\partial x^{2}}+x^{k+2} \frac{\partial}{\partial x^{1}}\right)-J_{2} \frac{\partial}{\partial x^{2 k+2}}\right), \ldots,\right. \\
& \left(x^{2 k+2}\left(\frac{\partial}{\partial x^{k+1}}+x^{2 k+1} \frac{\partial}{\partial x^{1}}\right)-J_{k+1} \frac{\partial}{\partial x^{2 k+2}}\right), \\
& \left.\left(\frac{\partial}{\partial x^{k+2}}-J_{k+2} \frac{\partial}{\partial x^{2 k+2}}\right), \ldots,\left(\frac{\partial}{\partial x^{2 k+1}}-J_{2 k+1} \frac{\partial}{\partial x^{2 k+2}}\right), \frac{\partial}{\partial x^{2 k+2}}, \ldots, \frac{\partial}{\partial x^{n}}\right) .
\end{aligned}
$$

Note that our choices of local coordinates were arbitrary up to at most functions of $(n-1)$ variables, while the local invariants $J_{1}, \ldots, J_{2 k+1}$ are arbitrary functions of $n$ variables. Therefore, point-affine distributions of this type locally depend essentially on $2 k+1$ arbitrary functions of $n$ variables.

Corollary 1. Let $\mathcal{F}$ be a rank 2 strictly affine point-affine distribution of constant type on a 3-dimensional manifold $X$.

1. If $\mathcal{F}$ is almost bracket-generating, then in a sufficiently small neighborhood of any point $x \in \mathcal{X}$, there exist local coordinates $\left(x^{1}, x^{2}, x^{3}\right)$ such that

$$
\mathcal{F}=\frac{\partial}{\partial x^{1}}+\operatorname{span}\left(\frac{\partial}{\partial x^{2}}, \frac{\partial}{\partial x^{3}}\right) \text {. }
$$

2. If $\mathcal{F}$ is bracket-generating and $L_{\mathcal{F}}$ is Frobenius, then in a sufficiently small neighborhood of any point $x \in \mathcal{X}$, there exist local coordinates $\left(x^{1}, x^{2}, x^{3}\right)$ such that

$$
\mathcal{F}=\left(x^{2} \frac{\partial}{\partial x^{1}}-J_{1} \frac{\partial}{\partial x^{2}}\right)+\operatorname{span}\left(\frac{\partial}{\partial x^{2}}, \frac{\partial}{\partial x^{3}}\right),
$$

where $J_{1}$ is an arbitrary function on $X$.

3. If $\mathcal{F}$ is bracket-generating and $L_{\mathcal{F}}$ is a contact distribution, then in a sufficiently small neighborhood of any point $x \in X$, there exist local coordinates $\left(x^{1}, x^{2}, x^{3}\right)$ such that

$$
\mathcal{F}=\left(\left(1+x^{3} J_{3}\right) \frac{\partial}{\partial x^{1}}+J_{3} \frac{\partial}{\partial x^{2}}-J_{2} \frac{\partial}{\partial x^{3}}\right)+\operatorname{span}\left(x^{3} \frac{\partial}{\partial x^{1}}+\frac{\partial}{\partial x^{2}}, \frac{\partial}{\partial x^{3}}\right),
$$

where $J_{2}, J_{3}$ are arbitrary functions on $X$.

Proof. Let $\bar{\eta}^{1}$ be as in Theorem 3.3.

1. If $\mathcal{F}$ is almost bracket-generating, then $d \bar{\eta}^{1}=0$, so $k=0$ and Case 1 of Theorem 3.3 applies.

2. If $\mathcal{F}$ is bracket-generating and $L_{\mathcal{F}}$ is Frobenius, then

$$
\bar{\eta}^{1} \wedge d \bar{\eta}^{1}=0, \quad d \bar{\eta}^{1} \neq 0,
$$

so $k=0$ and Case 2 of Theorem 3.3 applies.

3. If $\mathcal{F}$ is bracket-generating and $L_{\mathcal{F}}$ is a contact distribution, then

$$
\bar{\eta}^{1} \wedge d \bar{\eta}^{1} \neq 0, \quad\left(d \bar{\eta}^{1}\right)^{2}=0,
$$

so $k=1$ and Case 1 of Theorem 3.3 applies. 


\subsection{Equivalence problem for rank 1 point-affine distributions on 3-manifolds}

In this section we consider point-affine equivalence for rank 1 point-affine distributions $\mathcal{F}$ on 3-dimensional manifolds. We will assume that $\mathcal{F}$ is bracket-generating or almost bracketgenerating, since otherwise the equivalence problem may be reduced to a problem on a 2dimensional manifold.

Theorem 3.4. Let $\mathcal{F}$ be a rank 1 strictly affine point-affine distribution of constant type on a 3-dimensional manifold $X$.

1. If $\mathcal{F}$ is almost bracket-generating, then in a sufficiently small neighborhood of any point $x \in \mathcal{X}$, there exist local coordinates $\left(x^{1}, x^{2}, x^{3}\right)$ such that

$$
\mathcal{F}=\left(\frac{\partial}{\partial x^{1}}+x^{3} \frac{\partial}{\partial x^{2}}+J \frac{\partial}{\partial x^{3}}\right)+\operatorname{span}\left(\frac{\partial}{\partial x^{3}}\right),
$$

where $J$ is an arbitrary function on $\mathcal{X}$.

2. If $\mathcal{F}$ is bracket-generating and $L_{\mathcal{F}^{2}}$ (which must have rank 2 ) is Frobenius, then in a sufficiently small neighborhood of any point $x \in \mathcal{X}$, there exist local coordinates $\left(x^{1}, x^{2}, x^{3}\right)$ such that

$$
\mathcal{F}=\left(x^{2} \frac{\partial}{\partial x^{1}}+x^{3} \frac{\partial}{\partial x^{2}}+J \frac{\partial}{\partial x^{3}}\right)+\operatorname{span}\left(\frac{\partial}{\partial x^{3}}\right),
$$

where $J$ is an arbitrary function on $\mathcal{X}$.

3. If $\mathcal{F}$ is bracket-generating and $L_{\mathcal{F}^{2}}$ is not Frobenius, then in a sufficiently small neighborhood of any point $x \in \mathcal{X}$, there exist local coordinates $\left(x^{1}, x^{2}, x^{3}\right)$ such that

$$
\mathcal{F}=\left(\frac{\partial}{\partial x^{1}}+J\left(x^{3} \frac{\partial}{\partial x^{1}}+\frac{\partial}{\partial x^{2}}+H \frac{\partial}{\partial x^{3}}\right)\right)+\operatorname{span}\left(x^{3} \frac{\partial}{\partial x^{1}}+\frac{\partial}{\partial x^{2}}+H \frac{\partial}{\partial x^{3}}\right),
$$

where $H, J$ are arbitrary functions on $X$ satisfying $\frac{\partial H}{\partial x^{1}} \neq 0$.

Compare Theorem 3.4 with Theorem 2.6; in each case there is a new invariant $J$ which reflects the restriction that $v_{1}$ is a fixed vector field in $\mathcal{F}$.

Proof. A local framing of $\left(v_{1}, v_{2}, v_{3}\right)$ on $\mathcal{X}$ will be called admissible if $v_{1}$ is the distinguished vector field and

$$
\mathcal{F}=v_{1}+\operatorname{span}\left(v_{2}\right) .
$$

If $\left(v_{1}, v_{2}, v_{3}\right)$ is an admissible framing on $\mathcal{X}$, then any other admissible framing $\left(\tilde{v}_{1}, \tilde{v}_{2}, \tilde{v}_{3}\right)$ has the form

$$
\tilde{v}_{1}=v_{1}, \quad \tilde{v}_{2}=b_{2} v_{2}, \quad \tilde{v}_{3}=a_{3} v_{1}+b_{3} v_{2}+c_{3} v_{3},
$$

for some functions $a_{3}, b_{2}, b_{3}, c_{3}$ on $X$ which satisfy $b_{2} c_{3} \neq 0$.

A local coframing $\left(\bar{\eta}^{1}, \bar{\eta}^{2}, \bar{\eta}^{3}\right)$ on $\mathcal{X}$ will be called 0-adapted if it is the dual coframing of some admissible framing. Any two 0 -adapted coframings on $\mathcal{X}$ vary by a transformation of the form

$$
\left[\begin{array}{c}
\tilde{\bar{\eta}}^{1} \\
\tilde{\bar{\eta}}^{2} \\
\tilde{\bar{\eta}}^{3}
\end{array}\right]=\left[\begin{array}{ccc}
1 & 0 & a_{3} \\
0 & b_{2} & b_{3} \\
0 & 0 & c_{3}
\end{array}\right]^{-1}\left[\begin{array}{c}
\bar{\eta}^{1} \\
\bar{\eta}^{2} \\
\bar{\eta}^{3}
\end{array}\right],
$$

with $b_{2} c_{3} \neq 0$. 
The 0 -adapted coframings are the local sections of a principal fiber bundle $\mathcal{B}_{0} \rightarrow X$, with structure group $G_{0} \subset G L(3)$ consisting of all invertible matrices of the form in (3.15). The structure equations on $\mathcal{B}_{0}$ take the form

$$
\left[\begin{array}{l}
d \eta^{1} \\
d \eta^{2} \\
d \eta^{3}
\end{array}\right]=-\left[\begin{array}{ccc}
0 & 0 & \alpha_{3} \\
0 & \beta_{2} & \beta_{3} \\
0 & 0 & \gamma_{3}
\end{array}\right]\left[\begin{array}{c}
\eta^{1} \\
\eta^{2} \\
\eta^{3}
\end{array}\right]+\left[\begin{array}{c}
T_{12}^{1} \eta^{1} \wedge \eta^{2} \\
0 \\
T_{12}^{3} \eta^{1} \wedge \eta^{2}
\end{array}\right]
$$

Under a transformation of the form (3.15), the torsion functions $T_{12}^{1}, T_{12}^{3}$ transform as follows:

$$
\tilde{T}_{12}^{1}=b_{2} T_{12}^{1}-\frac{a_{3} b_{2}}{c_{3}} T_{12}^{3}, \quad \tilde{T}_{12}^{3}=\frac{b_{2}}{c_{3}} T_{12}^{3} .
$$

The function $T_{12}^{3}$ is a relative invariant, and the assumption that $\mathcal{F}$ is (almost) bracket-generating implies that $T_{12}^{3} \neq 0$. So, given any 0 -adapted coframing $\left(\bar{\eta}^{1}, \bar{\eta}^{2}, \bar{\eta}^{3}\right),(3.16)$ implies that we can perform a transformation of the form (3.15) to arrive at a new 0-adapted coframing for which $T_{12}^{3}=1, T_{12}^{1}=0$. Such a coframing will be called 1-adapted.

Any two 1-adapted coframings on $X$ vary by a transformation of the form

$$
\left[\begin{array}{c}
\tilde{\bar{\eta}}^{1} \\
\tilde{\bar{\eta}}^{2} \\
\tilde{\bar{\eta}}^{3}
\end{array}\right]=\left[\begin{array}{ccc}
1 & 0 & 0 \\
0 & b_{2} & b_{3} \\
0 & 0 & b_{2}
\end{array}\right]^{-1}\left[\begin{array}{c}
\bar{\eta}^{1} \\
\bar{\eta}^{2} \\
\bar{\eta}^{3}
\end{array}\right],
$$

with $b_{2} \neq 0$. The 1 -adapted coframings are the local sections of a principal fiber bundle $\mathcal{B}_{1} \subset \mathcal{B}_{0}$, with structure group $G_{1}$ consisting of all matrices of the form in (3.17). When restricted to $\mathcal{B}_{1}$, the connection forms $\alpha_{3}, \gamma_{3}-\beta_{2}$ become semi-basic, thereby introducing new torsion terms into the structure equations of $\mathcal{B}_{1}$. By adding multiples of the semi-basic forms to the connection forms $\beta_{2}, \beta_{3}$ so as to absorb as much of the torsion as possible, we can arrange that the structure equations of $\mathcal{B}_{1}$ take the form

$$
\left[\begin{array}{l}
d \eta^{1} \\
d \eta^{2} \\
d \eta^{3}
\end{array}\right]=-\left[\begin{array}{ccc}
0 & 0 & 0 \\
0 & \beta_{2} & \beta_{3} \\
0 & 0 & \beta_{2}
\end{array}\right]\left[\begin{array}{l}
\eta^{1} \\
\eta^{2} \\
\eta^{3}
\end{array}\right]+\left[\begin{array}{c}
\left(T_{13}^{1} \eta^{1}+T_{23}^{1} \eta^{2}\right) \wedge \eta^{3} \\
0 \\
\eta^{1} \wedge \eta^{2}+T_{13}^{3} \eta^{1} \wedge \eta^{3}
\end{array}\right]
$$

Under a transformation of the form (3.17), we have

$$
\tilde{T}_{13}^{1}=b_{2} T_{13}^{1}, \quad \tilde{T}_{23}^{1}=b_{2}^{2} T_{23}^{1}, \quad \tilde{T}_{13}^{3}=T_{13}^{3}+\frac{b_{3}}{b_{2}} .
$$

The functions $T_{13}^{1}, T_{23}^{1}$ are relative invariants; however, given any 1-adapted coframing $\left(\bar{\eta}^{1}, \bar{\eta}^{2}, \bar{\eta}^{3}\right)$, we can perform a transformation of the form (3.17) to arrive at a new 1-adapted coframing for which $T_{13}^{3}=0$. Such a coframing will be called 2-adapted.

Any two 2-adapted coframings on $X$ vary by a transformation of the form

$$
\left[\begin{array}{c}
\tilde{\bar{\eta}}^{1} \\
\tilde{\bar{\eta}}^{2} \\
\tilde{\bar{\eta}}^{3}
\end{array}\right]=\left[\begin{array}{ccc}
1 & 0 & 0 \\
0 & b_{2} & 0 \\
0 & 0 & b_{2}
\end{array}\right]^{-1}\left[\begin{array}{c}
\bar{\eta}^{1} \\
\bar{\eta}^{2} \\
\bar{\eta}^{3}
\end{array}\right],
$$

with $b_{2} \neq 0$. The 2 -adapted coframings are the local sections of a principal fiber bundle $\mathcal{B}_{2} \subset \mathcal{B}_{1}$, with structure group $G_{2}$ consisting of all matrices of the form in (3.18). When restricted to $\mathcal{B}_{2}$, the connection form $\beta_{3}$ becomes semi-basic, thereby introducing new torsion terms into the 
structure equations of $\mathcal{B}_{2}$. By adding multiples of the semi-basic forms to the connection form $\beta_{2}$ so as to absorb as much of the torsion as possible, we can arrange that the structure equations of $\mathcal{B}_{2}$ take the form

$$
\left[\begin{array}{l}
d \eta^{1} \\
d \eta^{2} \\
d \eta^{3}
\end{array}\right]=-\left[\begin{array}{ccc}
0 & 0 & 0 \\
0 & \beta_{2} & 0 \\
0 & 0 & \beta_{2}
\end{array}\right]\left[\begin{array}{l}
\eta^{1} \\
\eta^{2} \\
\eta^{3}
\end{array}\right]+\left[\begin{array}{c}
\left(T_{13}^{1} \eta^{1}+T_{23}^{1} \eta^{2}\right) \wedge \eta^{3} \\
T_{13}^{2} \eta^{1} \wedge \eta^{3} \\
\eta^{1} \wedge \eta^{2}
\end{array}\right]
$$

Under a transformation of the form (3.18), we have

$$
\tilde{T}_{13}^{1}=b_{2} T_{13}^{1}, \quad \tilde{T}_{23}^{1}=b_{2}^{2} T_{23}^{1}, \quad \tilde{T}_{13}^{2}=T_{13}^{2} .
$$

Thus the functions $T_{13}^{1}, T_{23}^{1}$ are relative invariants, while $T_{13}^{2}$ is a well-defined function on $X$. As in Section 3.1, we will divide into cases based on the vanishing/nonvanishing of $T_{23}^{1}$ and $T_{13}^{1}$.

Case 1. Suppose that $T_{23}^{1}=T_{13}^{1}=0$. Since $T_{13}^{2}$ is well-defined on $\mathcal{X}$, no further adaptations can be made based on the structure equations (3.19). The tableau consisting of all matrices of the form in (3.19) is not involutive, so in principle the next step in the method of equivalence would be to prolong the structure equations. However, equations (3.19) already contain enough information to produce a local normal form for such structures, so we will proceed in this direction.

Given any 1-adapted coframing, consider the dual framing $\left(v_{1}, v_{2}, v_{3}\right)$. The structure equations (3.19) imply that

$$
\left[v_{1}, v_{2}\right] \equiv-v_{3} \quad \bmod v_{2}, \quad\left[v_{2}, v_{3}\right] \equiv 0 \quad \bmod v_{2}, v_{3}, \quad\left[v_{1}, v_{3}\right] \equiv 0 \quad \bmod v_{2}, v_{3}
$$

The first equation implies that $L_{\mathcal{F}^{2}}$ is spanned by $\left\{v_{2}, v_{3}\right\}$; the second implies that $L_{\mathcal{F}^{2}}$ is a Frobenius distribution, and the third implies that $\mathcal{F}$ is almost bracket-generating. Thus, this case corresponds to Case 1 of Theorem 3.4.

In order to recover the normal form of Theorem 3.4, let $\left(\bar{\eta}^{1}, \bar{\eta}^{2}, \bar{\eta}^{3}\right)$ be any 1-adapted local coframing on $X$ given by a local section $\sigma: X \rightarrow \mathcal{B}_{2}$, and consider the pullbacks of the structure equations (3.19) via $\sigma$ :

$$
\begin{aligned}
& d \bar{\eta}^{1}=0, \\
& d \bar{\eta}^{2}=-\bar{\beta}_{2} \wedge \bar{\eta}^{2}+\bar{T}_{13}^{2} \bar{\eta}^{1} \wedge \bar{\eta}^{3}, \\
& d \bar{\eta}^{3}=-\bar{\beta}_{2} \wedge \bar{\eta}^{3}+\bar{\eta}^{1} \wedge \bar{\eta}^{2} .
\end{aligned}
$$

Since $\bar{\eta}^{1}$ is an exact 1 -form, we can choose a local coordinate $x^{1}$ on $X$ such that $\bar{\eta}^{1}=d x^{1}$. Then, since $\bar{\eta}^{3}$ is a contact form with

$$
d \bar{\eta}^{3} \equiv 0 \quad \bmod \left\{\bar{\eta}^{3}, d x^{1}\right\}
$$

there exist local coordinates $x^{2}, x^{3}$, functionally independent from $x^{1}$, and a nonvanishing function $\lambda$ on $X$ such that

$$
\bar{\eta}^{3}=\lambda\left(d x^{2}-x^{3} d x^{1}\right) .
$$

By replacing the given coframing with the (also 1-adapted) coframing $\left(\bar{\eta}^{1}, \lambda^{-1} \bar{\eta}^{2}, \lambda^{-1} \bar{\eta}^{3}\right)$, we can assume that $\lambda \equiv 1$. The third equation in (3.21) then implies that

$$
\bar{\eta}^{2}=d x^{3}+B d x^{1}+C\left(d x^{2}-x^{3} d x^{1}\right)
$$

for some functions $B, C$ on $X$, and that

$$
\bar{\beta}_{2}=C d x^{1}+D\left(d x^{2}-x^{3} d x^{1}\right)
$$


for some additional function $D$ on $\mathcal{X}$. Finally, the second equation in (3.21) implies that

$$
C=\frac{1}{2} B_{x^{3}}, \quad D=\frac{1}{2} B_{x^{3} x^{3}} .
$$

Our 2-adapted coframing now has the form

$$
\begin{aligned}
& \bar{\eta}^{1}=d x^{1}, \\
& \bar{\eta}^{2}=d x^{3}+B d x^{1}+\frac{1}{2} B_{x^{3}}\left(d x^{2}-x^{3} d x^{1}\right), \\
& \bar{\eta}^{3}=d x^{2}-x^{3} d x^{1} .
\end{aligned}
$$

The dual framing is

$$
v_{1}=\frac{\partial}{\partial x^{1}}+x^{3} \frac{\partial}{\partial x^{2}}-B \frac{\partial}{\partial x^{3}}, \quad v_{2}=\frac{\partial}{\partial x^{3}}, \quad v_{3}=\frac{\partial}{\partial x^{2}}-\frac{1}{2} B_{x^{3}} \frac{\partial}{\partial x^{3}}
$$

setting $J=-B$, we have:

$$
\mathcal{F}=v_{1}+\operatorname{span}\left(v_{2}\right)=\left(\frac{\partial}{\partial x^{1}}+x^{3} \frac{\partial}{\partial x^{2}}+J \frac{\partial}{\partial x^{3}}\right)+\operatorname{span}\left(\frac{\partial}{\partial x^{3}}\right) .
$$

Comparing the structure equations (3.21) with those of the coframing (3.22), we see that

$$
T_{13}^{2}=J_{x^{2}}-\frac{1}{2}\left(J_{x^{1} x^{3}}+x^{3} J_{x^{2} x^{3}}+J J_{x^{3} x^{3}}\right)+\frac{1}{4}\left(J_{x^{3}}\right)^{2} .
$$

Case 2. Suppose that $T_{23}^{1}=0, T_{13}^{1} \neq 0$. (3.20) implies that, given any 2-adapted coframing $\left(\bar{\eta}^{1}, \bar{\eta}^{2}, \bar{\eta}^{3}\right)$, we can perform a transformation of the form (3.18) to arrive at a new 2-adapted coframing for which $T_{13}^{1}=1$. Such a coframing will be called 3-adapted.

Any two 3 -adapted coframings on $X$ vary by a transformation of the form

$$
\left[\begin{array}{c}
\tilde{\bar{\eta}}^{1} \\
\tilde{\bar{\eta}}^{2} \\
\tilde{\bar{\eta}}^{3}
\end{array}\right]=\left[\begin{array}{ccc}
1 & 0 & 0 \\
0 & 1 & 0 \\
0 & 0 & 1
\end{array}\right]^{-1}\left[\begin{array}{c}
\bar{\eta}^{1} \\
\bar{\eta}^{2} \\
\bar{\eta}^{3}
\end{array}\right] .
$$

In other words, the 3 -adapted coframings are the local sections of an $(e)$-structure $\mathcal{B}_{3} \subset \mathcal{B}_{2}$. The projection $\pi: \mathcal{B}_{3} \rightarrow \mathcal{X}$ is a diffeomorphism, and there is a unique 3-adapted coframing $\left(\bar{\eta}^{1}, \bar{\eta}^{2}, \bar{\eta}^{3}\right)$ on $\mathcal{X}$.

When restricted to $\mathcal{B}_{3}$, the connection form $\beta_{2}$ becomes semi-basic. The first structure equation in (3.19) now takes the form

$$
d \eta^{1}=\eta^{1} \wedge \eta^{3}
$$

Differentiating this equation yields

$$
\beta_{2} \wedge \eta^{1} \wedge \eta^{3}=0
$$

therefore, $\beta_{2}$ is a linear combination of $\eta^{1}$ and $\eta^{3}$, and the structure equations of $\mathcal{B}_{3}$ take the form

$$
\begin{aligned}
& d \eta^{1}=\eta^{1} \wedge \eta^{3} \\
& d \eta^{2}=T_{12}^{2} \eta^{1} \wedge \eta^{2}+T_{13}^{2} \eta^{1} \wedge \eta^{3}+T_{23}^{2} \eta^{2} \wedge \eta^{3} \\
& d \eta^{3}=\eta^{1} \wedge \eta^{2}+T_{12}^{2} \eta^{1} \wedge \eta^{3} .
\end{aligned}
$$


Given any 3 -adapted coframing, consider the dual framing $\left(v_{1}, v_{2}, v_{3}\right)$. The structure equations (3.23) imply that

$$
\left[v_{1}, v_{2}\right] \equiv-v_{3} \quad \bmod v_{2}, \quad\left[v_{2}, v_{3}\right] \equiv 0 \quad \bmod v_{2}, \quad\left[v_{1}, v_{3}\right] \equiv-v_{1} \quad \bmod v_{2} .
$$

The first equation implies that $L_{\mathcal{F}^{2}}$ is spanned by $\left\{v_{2}, v_{3}\right\}$; the second implies that $L_{\mathcal{F}^{2}}$ is a Frobenius distribution, and the third implies that $\mathcal{F}$ is bracket-generating. Thus, this case corresponds to Case 2 of Theorem 3.4.

In order to recover the normal form of Theorem 3.4 , let $\left(\bar{\eta}^{1}, \bar{\eta}^{2}, \bar{\eta}^{3}\right)$ be the 3 -adapted local coframing on $X$ given by the unique local section $\sigma: \mathcal{X} \rightarrow \mathcal{B}_{3}$, and consider the pullbacks of the structure equations (3.23) via $\sigma$. Since $\bar{\eta}^{1}$ is an integrable 1 -form (i.e., $d \bar{\eta}^{1} \equiv 0 \bmod \bar{\eta}^{1}$ ) but not exact, we can choose local coordinates $x^{1}, x^{2}$ on $X$ such that $\bar{\eta}^{1}=\frac{1}{x^{2}} d x^{1}$. Then the first equation in (3.23) implies that

$$
\bar{\eta}^{3} \equiv \frac{1}{x^{2}} d x^{2} \quad \bmod d x^{1}
$$

Now, since $d \bar{\eta}^{3} \not \equiv 0 \bmod \bar{\eta}^{3}$, there must exist another local coordinate $x^{3}$, functionally independent from $x^{1}, x^{2}$, such that

$$
\bar{\eta}^{3}=\frac{1}{x^{2}}\left(d x^{2}-\frac{x^{3}}{x^{2}} d x^{1}\right) .
$$

Moreover, the third equation in (3.23) implies that

$$
\bar{\eta}^{2}=\frac{1}{x^{2}}\left(\left(d x^{3}+\frac{1}{x^{2}} B d x^{1}+\frac{1}{x^{2}} C\left(d x^{2}-\frac{x^{3}}{x^{2}} d x^{1}\right)\right)\right.
$$

for some functions $B, C$ on $X$. Finally, the second equation in (3.23) implies that

$$
C=\frac{1}{2}\left(x^{2} B_{x^{3}}-x^{3}\right) .
$$

Our 3-adapted coframing now has the form

$$
\begin{aligned}
& \bar{\eta}^{1}=\frac{1}{x^{2}} d x^{1} \\
& \bar{\eta}^{2}=\frac{1}{x^{2}} d x^{3}+\frac{1}{\left(x^{2}\right)^{2}} B d x^{1}+\frac{1}{2}\left(\frac{\left(x^{2} B_{x^{3}}-x^{3}\right)}{\left(x^{2}\right)^{2}}\right)\left(d x^{2}-\frac{x^{3}}{x^{2}} d x^{1}\right) \\
& \bar{\eta}^{3}=\frac{1}{x^{2}} d x^{2}-\frac{x^{3}}{\left(x^{2}\right)^{2}} d x^{1} .
\end{aligned}
$$

The dual framing is

$$
\begin{aligned}
& v_{1}=x^{2} \frac{\partial}{\partial x^{1}}+x^{3} \frac{\partial}{\partial x^{2}}-B \frac{\partial}{\partial x^{3}} \\
& v_{2}=x^{2} \frac{\partial}{\partial x^{3}}, \\
& v_{3}=x^{2} \frac{\partial}{\partial x^{2}}-\frac{1}{2}\left(x^{2} B_{x^{3}}-x^{3}\right) \frac{\partial}{\partial x^{3}} ;
\end{aligned}
$$

setting $J=-B$ and noting that $\operatorname{span}\left(v_{2}\right)=\operatorname{span}\left(\frac{\partial}{\partial x^{3}}\right)$, we have:

$$
\mathcal{F}=v_{1}+\operatorname{span}\left(v_{2}\right)=\left(x^{2} \frac{\partial}{\partial x^{1}}+x^{3} \frac{\partial}{\partial x^{2}}+J \frac{\partial}{\partial x^{3}}\right)+\operatorname{span}\left(\frac{\partial}{\partial x^{3}}\right) .
$$


Comparing the structure equations (3.23) with those of the coframing (3.24), we see that

$$
\begin{aligned}
T_{12}^{2}= & \frac{1}{2 x^{2}}\left(x^{2} J_{x^{3}}-3 x^{3}\right), \\
T_{13}^{2}= & \frac{1}{4\left(x^{2}\right)^{2}}\left(3\left(x^{3}\right)^{2}-6 x^{2} J+4\left(x^{2}\right)^{2} J_{x^{2}}+2 x^{2} x^{3} J_{x^{3}}-2\left(x^{2}\right)^{3} J_{x^{1} x^{3}}\right. \\
& \left.-2\left(x^{2}\right)^{2} x^{3} J_{x^{2} x^{3}}+\left(x^{2}\right)^{2}\left(J_{x^{3}}\right)^{2}-2\left(x^{2}\right)^{2} J J_{x^{3} x^{3}}\right), \\
T_{23}^{2}= & \frac{1}{2}\left(1-x^{2} J_{x^{3} x^{3}}\right) .
\end{aligned}
$$

Case 3. Suppose that $T_{23}^{1} \neq 0 . \quad$ (3.20) implies that, given any 2-adapted coframing $\left(\bar{\eta}^{1}, \bar{\eta}^{2}, \bar{\eta}^{3}\right)$, we can perform a transformation of the form (3.18) to arrive at a new 1-adapted coframing for which $T_{23}^{1}= \pm 1$. Such a coframing will be called 3-adapted. (For ease of notation, let $\varepsilon=T_{23}^{1}= \pm 1$.)

Any two 3 -adapted coframings on $\mathcal{X}$ vary by a transformation of the form

$$
\left[\begin{array}{c}
\tilde{\bar{\eta}}^{1} \\
\tilde{\bar{\eta}}^{2} \\
\tilde{\bar{\eta}}^{3}
\end{array}\right]=\left[\begin{array}{ccc}
1 & 0 & 0 \\
0 & \pm 1 & 0 \\
0 & 0 & \pm 1
\end{array}\right]^{-1}\left[\begin{array}{c}
\bar{\eta}^{1} \\
\bar{\eta}^{2} \\
\bar{\eta}^{3}
\end{array}\right],
$$

where the signs are chosen such that the matrix above has determinant equal to 1 . The corresponding principal fiber bundle $\mathcal{B}_{3} \subset \mathcal{B}_{2}$ has discrete fiber group $G_{3} \cong \mathbb{Z} / 2 \mathbb{Z}$, and we can regard $\mathcal{B}_{3}$ as an (e)-structure over a 2 -to- 1 cover of $\mathcal{X}$. When restricted to $\mathcal{B}_{3}$, the connection form $\beta_{2}$ becomes semi-basic; the structure equations of $\mathcal{B}_{3}$ take the form

$$
\begin{aligned}
& d \eta^{1}=T_{13}^{1} \eta^{1} \wedge \eta^{3}+\varepsilon \eta^{2} \wedge \eta^{3}, \\
& d \eta^{2}=T_{12}^{2} \eta^{1} \wedge \eta^{2}+T_{13}^{2} \eta^{1} \wedge \eta^{3}+T_{23}^{2} \eta^{2} \wedge \eta^{3}, \\
& d \eta^{3}=\eta^{1} \wedge \eta^{2}+T_{12}^{2} \eta^{1} \wedge \eta^{3}+T_{23}^{3} \eta^{2} \wedge \eta^{3} .
\end{aligned}
$$

Given any 3 -adapted coframing, consider the dual framing $\left(v_{1}, v_{2}, v_{3}\right)$. The structure equations (3.25) imply that

$$
\left[v_{1}, v_{2}\right] \equiv-v_{3} \quad \bmod v_{2}, \quad\left[v_{2}, v_{3}\right] \equiv-\varepsilon v_{1} \quad \bmod v_{2}, v_{3}, \quad\left[v_{1}, v_{3}\right] \equiv 0 \quad \bmod v_{1}, v_{2}, v_{3} .
$$

The first equation implies that $L_{\mathcal{F}^{2}}$ is spanned by $\left\{v_{2}, v_{3}\right\}$, and the second implies that $L_{\mathcal{F}^{2}}$ is not Frobenius and that $\mathcal{F}$ is bracket-generating. Thus, this case corresponds to Case 3 of Theorem 3.4.

In order to recover the normal form of Theorem 3.4 , let $\left(\bar{\eta}^{1}, \bar{\eta}^{2}, \bar{\eta}^{3}\right)$ be any 3 -adapted local coframing on $\mathcal{X}$ given by a local section $\sigma: \mathcal{X} \rightarrow \mathcal{B}_{3}$, and consider the pullbacks of the structure equations (3.25) via $\sigma$. Since $d \bar{\eta}^{1} \not \equiv 0 \bmod \bar{\eta}^{1}$, Pfaff's theorem implies that we can choose local coordinates $x^{1}, x^{2}, x^{3}$ on $X$ such that

$$
\bar{\eta}^{1}=d x^{1}-x^{3} d x^{2} .
$$

Set $T_{13}^{1}=B$; this will simplify notation in what follows. The first equation in (3.25) becomes

$$
\left(B \bar{\eta}^{1}+\varepsilon \bar{\eta}^{2}\right) \wedge \bar{\eta}^{3}=d x^{2} \wedge d x^{3},
$$

which implies that

$$
\begin{aligned}
& \bar{\eta}^{2}=\varepsilon\left(-B\left(d x^{1}-x^{3} d x^{2}\right)-\lambda^{-1} d x^{2}+C\left(H d x^{2}-d x^{3}\right)\right), \\
& \bar{\eta}^{3}=\lambda\left(H d x^{2}-d x^{3}\right),
\end{aligned}
$$


for some functions $\lambda, C, H$ on $X$ with $\lambda \neq 0$. (If necessary, perform the contact transformation

$$
x^{1} \rightarrow x^{1}-x^{2} x^{3}, \quad x^{2} \rightarrow x^{3}, \quad x^{3} \rightarrow-x^{2}
$$

to achieve this form.) Now the third equation in (3.25) implies that

$$
-\varepsilon \lambda^{2} H_{x^{1}}=1 \text {. }
$$

Therefore, $H_{x^{1}} \neq 0 ; \varepsilon H_{x^{1}}<0$, and

$$
\lambda= \pm \frac{1}{\sqrt{-\varepsilon H_{x^{1}}}} .
$$

The $\mathbb{Z} / 2 \mathbb{Z}$ freedom in the structure group $G_{3}$ may be used to eliminate the sign ambiguity in $\lambda$; for simplicity we will take the positive square root.

Our 3-adapted coframing now has the form

$$
\begin{aligned}
& \bar{\eta}^{1}=d x^{1}-x^{3} d x^{2}, \\
& \bar{\eta}^{2}=-\varepsilon\left(\sqrt{-\varepsilon H_{x^{1}}} d x^{2}+B\left(d x^{1}-x^{3} d x^{2}\right)+C\left(H d x^{2}-d x^{3}\right)\right), \\
& \bar{\eta}^{3}=\frac{1}{\sqrt{-\varepsilon H_{x^{1}}}}\left(H d x^{2}-d x^{3}\right) .
\end{aligned}
$$

The dual framing is

$$
\begin{aligned}
& v_{1}=\frac{\partial}{\partial x^{1}}-\frac{B}{\sqrt{-\varepsilon H_{x^{1}}}}\left(x^{3} \frac{\partial}{\partial x^{1}}+\frac{\partial}{\partial x^{2}}+H \frac{\partial}{\partial x^{3}}\right), \\
& v_{2}=-\frac{\varepsilon}{\sqrt{-\varepsilon H_{x^{1}}}}\left(x^{3} \frac{\partial}{\partial x^{1}}+\frac{\partial}{\partial x^{2}}+H \frac{\partial}{\partial x^{3}}\right), \\
& v_{3}=-\sqrt{-\varepsilon H_{x^{1}}} \frac{\partial}{\partial x^{3}}+C\left(x^{3} \frac{\partial}{\partial x^{1}}+\frac{\partial}{\partial x^{2}}+H \frac{\partial}{\partial x^{3}}\right) ;
\end{aligned}
$$

setting $J=\frac{-B}{\sqrt{-\varepsilon H_{x^{1}}}}$, we have:

$$
\begin{aligned}
\mathcal{F} & =v_{1}+\operatorname{span}\left(v_{2}\right) \\
& =\left(\frac{\partial}{\partial x^{1}}+J\left(x^{3} \frac{\partial}{\partial x^{1}}+\frac{\partial}{\partial x^{2}}+H \frac{\partial}{\partial x^{3}}\right)\right)+\operatorname{span}\left(x^{3} \frac{\partial}{\partial x^{1}}+\frac{\partial}{\partial x^{2}}+H \frac{\partial}{\partial x^{3}}\right) .
\end{aligned}
$$

Comparing the structure equations (3.25) with those of the coframing (3.26) shows that

$$
\begin{aligned}
C=\frac{1}{2 H_{x^{1}}} \sqrt{-\varepsilon H_{x^{1}}} & \left(H_{x^{1}} H_{x^{3}}-H H_{x^{1} x^{3}}-H_{x^{1} x^{2}}\right) J \\
& \left.-\left(1+x^{3} J\right) H_{x^{1} x^{1}}-\left(J_{x^{2}}+x^{3} J_{x^{1}}+H J_{x^{3}}\right) H_{x^{1}}\right),
\end{aligned}
$$

and that

$$
\begin{aligned}
T_{12}^{2}= & \frac{1}{2}\left(J_{x^{2}}+x^{3} J_{x^{1}}+H J_{x^{3}}+J H_{x^{3}}\right) \\
T_{13}^{2}= & \varepsilon C^{2} H_{x^{1}}-H_{x^{1}} J_{x^{3}}+J^{2} H_{x^{1}}+\frac{1}{2 \sqrt{-\varepsilon H_{x^{1}}}}\left(2 C \left(H_{x^{1}} J_{x^{2}}+J H_{x^{1}, x^{2}}+H_{x^{1} x^{1}}\right.\right. \\
& \left.+2 x^{3} H_{x^{1}} J_{x^{1}}+x^{3} J H_{x^{1} x^{1}}+2 H H_{x^{1}} J_{x^{3}}+H H_{x^{1} x^{3}} J-2 H_{x^{1}} H_{x^{3}} J\right) \\
& \left.-2 x^{3} C_{x^{1}} H_{x^{1}} J-2 C_{x^{1}} H_{x^{1}}-2 C_{x^{3}} H H_{x^{1}} J-2 C_{x^{2}} H_{x^{1}} J\right) \\
T_{23}^{2}= & -\left(C_{x^{2}}+x^{3} C_{x^{1}}+H C_{x^{3}}+C H_{x^{3}}\right)-\frac{\varepsilon}{2 \sqrt{-\varepsilon H_{x^{1}}}}\left(H_{x^{1} x^{3}}+2 J H_{x^{1}}\right), \\
T_{23}^{3}= & \frac{\varepsilon}{2 H_{x^{1}} \sqrt{-\varepsilon H_{x^{1}}}}\left(x^{3} H_{x^{1} x^{1}}-2 H_{x^{1}} H_{x^{3}}+H_{x^{1} x^{2}}+H H_{x^{1} x^{3}}\right) .
\end{aligned}
$$




\section{Examples and questions for further study}

\subsection{Examples}

Example 4.1 (NMR control). In quantum mechanics, a particle with spin $\frac{1}{2}$ is represented by a state vector

$$
|\psi\rangle=\left[\begin{array}{l}
\alpha \\
\beta
\end{array}\right] \in \mathbb{C}^{2}
$$

with $|\alpha|^{2}+|\beta|^{2}=1$. In the presence of a magnetic field $\mathbf{B}=B_{x} \mathbf{i}+B_{y} \mathbf{j}+B_{z} \mathbf{k}$, the state vector at time $t$ is

$$
|\psi(t)\rangle=U(t)\left|\psi_{0}\right\rangle
$$

where $U: \mathbb{R} \rightarrow S U(2)$ is a solution of Schrödinger's equation

$$
\dot{U}=-i \mathcal{H} U \text {. }
$$

(See [22] for more details.) The Hamiltonian operator $\mathcal{H}$ is given by

$$
\mathcal{H}=-\gamma \hbar\left(B_{x} \cdot I_{x}+B_{y} \cdot I_{y}+B_{z} \cdot I_{z}\right),
$$

where $\gamma$ is the gyromagnetic ratio (a constant), $\hbar=\frac{h}{2 \pi}$ (where $h$ is Planck's constant), and $I_{x}, I_{y}$, and $I_{z}$ are the Pauli spin matrices

$$
I_{x}=\frac{1}{2}\left[\begin{array}{ll}
0 & 1 \\
1 & 0
\end{array}\right], \quad I_{y}=\frac{1}{2}\left[\begin{array}{cc}
0 & -i \\
i & 0
\end{array}\right], \quad I_{z}=\frac{1}{2}\left[\begin{array}{cc}
1 & 0 \\
0 & -1
\end{array}\right] .
$$

By multiplying the Pauli spin matrices by $-i$ as in (4.1) we obtain members of the Lie algebra of $S U(2)$. The commutation relations for $-i I_{x},-i I_{y}$, and $-i I_{z}$ are

$$
\left[-i I_{x},-i I_{y}\right]=-i I_{z}, \quad\left[-i I_{y},-i I_{z}\right]=-i I_{x}, \quad\left[-i I_{z},-i I_{x}\right]=-i I_{y} .
$$

In nuclear magnetic resonance (NMR) experiments, an ensemble of atomic nuclei of the same type can be modeled as a single spin system [14]. In such experiments, an ensemble of nuclei is subjected to a constant magnetic field $\mathbf{B}_{\mathbf{0}}$ in the $z$-direction (called the longitudinal field), and an oscillating field $\mathbf{B}_{\mathbf{1}}$ in the $x y$-plane (called the $r f$ field):

$$
\begin{aligned}
& \mathbf{B}_{\mathbf{0}}=B_{0} \mathbf{k}, \\
& \mathbf{B}_{\mathbf{1}}=B_{1}(\cos (\omega t+\phi) \mathbf{i}+\sin (\omega t+\phi) \mathbf{j}),
\end{aligned}
$$

where $\omega$ is the frequency of the rf field and $\phi$ is a phase. The Hamiltonian $\mathcal{H}$ can thus be written as

$$
\mathcal{H}=-\gamma \hbar\left(B_{0} \cdot I_{z}+B_{1}\left(\cos (\omega t+\phi) I_{x}+\sin (\omega t+\phi) I_{y}\right) .\right.
$$

In NMR experiments, the magnitude of the longitudinal field $\mathbf{B}_{\mathbf{0}}$ is usually kept constant, while the magnitude of the rf field $\mathbf{B}_{\mathbf{1}}$ may be used as a control variable. By transforming the coordinate system to a system that rotates at the same frequency $\omega$ as the rf field, and normalizing the constant $-\gamma \hbar B_{0}$ to 1 , we may rewrite the Hamiltonian as

$$
\mathcal{H}=I_{z}+u(t) I_{x},
$$

where $u(t)=\frac{B_{1}(t)}{B_{0}}$. 
Thus Schrödinger's equation (4.1) becomes the control-affine system

$$
\dot{U}=-i I_{z} U-\left(i I_{x} U\right) u
$$

on the 3-dimensional state space $S U(2)$. This system corresponds to a strictly affine, rank 1 point-affine distribution

$$
\mathcal{F}=v_{1}+\operatorname{span}\left(v_{2}\right)
$$

where $v_{1}, v_{2}$ are the right-invariant vector fields

$$
v_{1}=-i I_{z} U, \quad v_{2}=-i I_{x} U
$$

on $S U(2)$. Let $\alpha_{1}, \alpha_{2}, \alpha_{3}$ denote the canonical right-invariant 1-forms on $S U(2)$ dual to the basis $-i I_{x} U,-i I_{y} U,-i I_{z} U$ for the right-invariant vector fields; these forms satisfy the structure equations

$$
d \alpha_{1}=\alpha_{2} \wedge \alpha_{3}, \quad d \alpha_{2}=\alpha_{3} \wedge \alpha_{1}, \quad d \alpha_{3}=\alpha_{1} \wedge \alpha_{2}
$$

A 0 -adapted coframing for the system (4.2) (corresponding to the choice $v_{3}=-\left[v_{1}, v_{2}\right]=-i I_{y} U$ ) is given by

$$
\eta^{1}=\alpha_{3}, \quad \eta^{2}=\alpha_{1}, \quad \eta^{3}=\alpha_{2} .
$$

This coframing has structure equations

$$
d \eta^{1}=\eta^{2} \wedge \eta^{3}, \quad d \eta^{2}=\eta^{3} \wedge \eta^{1}, \quad d \eta^{3}=\eta^{1} \wedge \eta^{2}
$$

therefore this example falls into Case 3 of Theorem 3.4, with $\varepsilon=1$. Since $T_{13}^{1}=0$, we have $J=0$.

Let $x^{1}, x^{2}, x^{3}$ be local coordinates such that $\eta^{1}=d x^{1}-x^{3} d x^{2}$. Then as in the proof of Theorem 3.4, there exist functions $C, H, \lambda$ such that

$$
\begin{aligned}
& \left.\bar{\eta}^{2}=-\lambda^{-1} d x^{2}+C\left(H d x^{2}-d x^{3}\right)\right), \\
& \bar{\eta}^{3}=\lambda\left(H d x^{2}-d x^{3}\right) .
\end{aligned}
$$

(Note that $B=0$ in this example.) The structure equations imply that

$$
\begin{aligned}
& H=-e^{2 f\left(x^{2}, x^{3}\right)} \tan \left(x^{1}+g\left(x^{2}, x^{3}\right)\right)+h\left(x^{2}, x^{3}\right), \\
& C=-e^{-f\left(x^{2}, x^{3}\right)} \sin \left(x^{1}+g\left(x^{2}, x^{3}\right)\right), \\
& \lambda=e^{-f\left(x^{2}, x^{3}\right)} \cos \left(x^{1}+g\left(x^{2}, x^{3}\right)\right),
\end{aligned}
$$

for functions $f, g, h$ which satisfy the additional PDEs

$$
\begin{aligned}
& e^{2 f} f_{x^{3}}+g_{x^{2}}+h g_{x^{3}}+x^{3}=0, \\
& e^{2 f} g_{x^{3}}+f_{x^{2}}+h f_{x^{3}}-h_{x^{3}}=0 .
\end{aligned}
$$

Thus the invariants for this system are

$$
H=-e^{2 f\left(x^{2}, x^{3}\right)} \tan \left(x^{1}+g\left(x^{2}, x^{3}\right)\right)+h\left(x^{2}, x^{3}\right), \quad J=0 .
$$




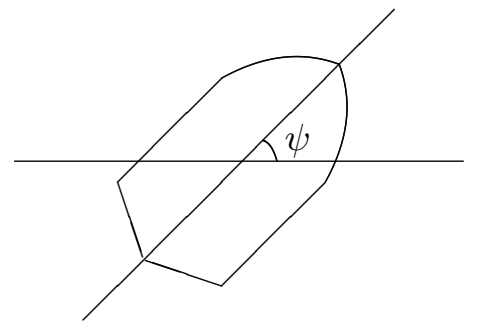

Figure 1. Heading angle $\psi$ in Example 4.2.

Example 4.2 (Navigation on a river). Consider a boat navigating on a river with a current; for simplicity, assume that the current runs parallel to the $x$-axis with constant speed $c$. The state of the boat is represented by its position $(x, y)$ and heading angle $\psi$ (see Fig. 1); thus the state space is $X=\mathbb{R}^{2} \times S^{1}$.

Due to asymmetry in the shape of the boat's hull, the current may have a rotational effect as well as a translational effect on the boat, and this effect depends on the boat's heading angle. Thus we will assume that the drift vector field has the form

$$
v_{1}=c \frac{\partial}{\partial x}+r(\psi) \frac{\partial}{\partial \psi} \text {. }
$$

We also assume that the boat can be propelled in a forward or backward direction, corresponding to the vector field

$$
v_{2}=(\cos \psi) \frac{\partial}{\partial x}+(\sin \psi) \frac{\partial}{\partial y},
$$

and that it can be steered to the right or left, corresponding to the vector field

$$
v_{3}=\frac{\partial}{\partial \psi} \text {. }
$$

The navigation problem for the boat is then the control-affine system corresponding to the rank 2 point-affine distribution

$$
\mathcal{F}=v_{1}+\operatorname{span}\left(v_{2}, v_{3}\right)
$$

on $X$.

Note that $\mathcal{F}$ fails to be strictly affine precisely when $\psi=0$ or $\psi=\pi$, since then $v_{1}$ is contained in $L_{\mathcal{F}}$. Thus our results are only applicable when the boat is not pointed directly upstream or downstream. Since $L_{\mathcal{F}}=\operatorname{span}\left(v_{2}, v_{3}\right)$ is a contact distribution, this system falls into Case 3 of Corollary 1.

The dual coframing to the framing $\left(v_{1}, v_{2}, v_{3}\right)$ on $\mathcal{X}$ is

$$
\bar{\eta}^{1}=\frac{1}{c}(d x-(\cot \psi) d y), \quad \bar{\eta}^{2}=(\csc \psi) d y, \quad \bar{\eta}^{3}=d \psi-\frac{r(\psi)}{c}(d x-(\cot \psi) d y) .
$$

This coframing has

$$
d \bar{\eta}^{1} \equiv-\frac{(\csc \psi)}{c} \bar{\eta}^{2} \wedge \bar{\eta}^{3} \bmod \bar{\eta}^{1}
$$

in order to obtain a 0 -adapted coframing as in the proof of Theorem 3.3, we multiply $\bar{\eta}^{2}$ by $\frac{(\sin \psi)}{c}$ and $\bar{\eta}^{3}$ by $-\left(\csc ^{2} \psi\right)$, resulting in the following coframing:

$$
\begin{aligned}
& \bar{\eta}^{1}=\frac{1}{c}(d x-(\cot \psi) d y), \quad \bar{\eta}^{2}=\frac{1}{c} d y, \\
& \bar{\eta}^{3}=-\left(\csc ^{2} \psi\right)\left(d \psi-\frac{r(\psi)}{c}(d x-(\cot \psi) d y)\right) .
\end{aligned}
$$


The local coordinate functions

$$
x^{1}=\frac{x}{c}, \quad x^{2}=\frac{y}{c}, \quad x^{3}=\cot \psi
$$

are Pfaff normal form coordinates for $\bar{\eta}^{1}$; i.e.,

$$
\bar{\eta}^{1}=d x^{1}-x^{3} d x^{2} .
$$

In terms of these coordinates, the coframing (4.3) becomes:

$$
\bar{\eta}^{1}=d x^{1}-x^{3} d x^{2}, \quad \bar{\eta}^{2}=d x^{2}, \quad \bar{\eta}^{3}=d x^{3}-\left(\left(x^{3}\right)^{2}+1\right) R\left(x^{3}\right)\left(d x^{1}-x^{3} d x^{2}\right),
$$

where $R\left(x^{3}\right)=r(\psi)=r\left(\cot ^{-1}\left(x^{3}\right)\right)$. Thus the invariants for this system are

$$
J_{2}=\left(\left(x^{3}\right)^{2}+1\right) R\left(x^{3}\right)=\left(\csc ^{2} \psi\right) r(\psi), \quad J_{3}=0 .
$$

\subsection{Questions for further study}

There are two main issues that we hope to address in future papers, both motivated by optimal control theory.

1. What metric structures (analogous to sub-Riemannian or sub-Finsler geometry for linear distributions) are appropriate for point-affine distributions, and what can we say about their geometry, geodesics, etc.? Even for linear distributions, issues such as controllability and the presence of rigid curves - i.e., curves with no $C^{1}$ variations whatsoever among horizonal curves - are nontrivial, and the study of optimal trajectories for sub-Riemannian and sub-Finsler metrics is quite complicated. (See, for instance, [20, 21, 9].) Agrachev and Sarychev have studied time-optimal extremals and given necessary and sufficient conditions for ridigity of trajectories for affine distributions in [2], but the consequences of imposing any type of metric structure on a point-affine distribution and the resulting geometry remain entirely unexplored. These issues are obviously important for understanding the associated control problems.

2. What can we say about the geometry of affine distributions of non-constant type, particularly for affine distributions which are not strictly affine? Normal forms for non-strictly affine distributions of rank 1 have been given by Kang and Krener [19], Kang [18], and Tall and Respondek [24], and for corank-1 affine distributions by Jakubczyk and Respondek [17], Respondek [23], and Zhitomirskii and Respondek [26]. However, intermediate ranks are important as well - for instance, for understanding second-order systems arising in mechanics, e.g.

$$
\ddot{x}=\sum_{i=1}^{s} u^{i} F_{i}(x) .
$$

This system can be rewritten as the following first-order system on the cotangent bundle $T^{*} \mathcal{X}$, with local coordinates $(x, p)$ :

$$
\begin{aligned}
& \dot{x}=p, \\
& \dot{p}=\sum_{i=1}^{s} u^{i} F_{i}(x) .
\end{aligned}
$$

The associated rank $s$ affine distribution fails to be strictly affine precisely along the submanifold $\{p=0\}$ of stationary points. The behavior of trajectories near such points is of vital interest in control theory, so it would be useful to understand the geometry of such structures. Elkin has introduced the notion of a " $t$-codistribution" in $[12,13]$, and we anticipate that this will be a useful tool for extending our methods to affine distributions of this type. 


\section{Acknowledgements}

This work was partially supported by NSF grant DMS-0908456.

\section{References}

[1] Agrachev A.A., Feedback-invariant optimal control theory and differential geometry. II. Jacobi curves for singular extremals, J. Dynam. Control Systems 4 (1998), 583-604.

[2] Agrachev A.A., Sarychev A.V., Abnormal sub-Riemannian geodesics: Morse index and rigidity, Ann. Inst. H. Poincaré Anal. Non Linéaire 13 (1996), 635-690.

[3] Agrachev A., Zelenko I., On feedback classification of control-affine systems with one- and two-dimensional inputs, SIAM J. Control Optim. 46 (2007), 1431-1460, math.OC/0502031.

[4] Bryant R.L., Chern S.S., Gardner R.B., Goldschmidt H.L., Griffiths P.A., Exterior differential systems, Mathematical Sciences Research Institute Publications, Vol. 18, Springer-Verlag, New York, 1991.

[5] Bryant R.L., Conformal geometry and 3-plane fields on 6-manifolds, in Developments of Cartan Geometry and Related Mathematical Problems, RIMS Symposium Proceedings, Vol. 1502, Kyoto University, 2006, 1-15, math.DG/0511110.

[6] Bullo F., Lewis A.D., Geometric control of mechanical systems. Modeling, analysis, and design for simple mechanical control systems, Texts in Applied Mathematics, Vol. 49, Springer-Verlag, New York, 2005.

[7] Cartan É., Les systèmes de Pfaff, à cinq variables et les équations aux dérivées partielles du second ordre, Ann. Sci. École Norm. Sup. (3) 27 (1910), 109-192.

[8] Clelland J.N., Moseley C.G., Sub-Finsler geometry in dimension three, Differential Geom. Appl. 24 (2006), 628-651, math.DG/0406439.

[9] Clelland J.N., Moseley C.G., Wilkens G.R., Geometry of sub-Finsler Engel manifolds, Asian J. Math. 11 (2007), 699-726.

[10] Doubrov B., Zelenko I., On local geometry of nonholonomic rank 2 distributions, math.DG/0703662.

[11] Doubrov B., Zelenko I., On local geometry of rank 3 distributions with 6-dimensional square, arXiv:0807.3267.

[12] Elkin V.I., Affine control systems: their equivalence, classification, quotient systems, and subsystems. Optimization and control, 1, J. Math. Sci. (New York) 88 (1998), 675-721.

[13] Elkin V.I., Reduction of nonlinear control systems. A differential geometric approach, Mathematics and its Applications, Vol. 472, Kluwer Academic Publishers, Dordrecht, 1999.

[14] Ernst R., Bodenhausen G., Wokaun A., Principles of nuclear magnetic resonance in one and two dimensions, Oxford University Press, Oxford, 1987.

[15] Gardner R.B., The method of equivalence and its applications, CBMS-NSF Regional Conference Series in Applied Mathematics, Vol. 58, Society for Industrial and Applied Mathematics (SIAM), Philadelphia, PA, 1989.

[16] Ivey T.A., Landsberg J.M., Cartan for beginners: differential geometry via moving frames and exterior differential systems, Graduate Studies in Mathematics, Vol. 61, American Mathematical Society, Providence, RI, 2003.

[17] Jakubczyk B., Respondek W., Feedback classification of analytic control systems in the plane, in Analysis of Controlled Dynamical Systems (Lyon, 1990), Progr. Systems Control Theory, Vol. 8, Birkhäuser Boston, Boston, MA, 1991, 263-273.

[18] Kang W., Extended controller form and invariants of nonlinear control systems with a single input, J. Math. Systems Estim. Control 6 (1996), 27-51.

[19] Kang W., Krener A.J., Extended quadratic controller normal form and dynamic state feedback linearization of nonlinear systems, SIAM J. Control Optim. 30 (1992), 1319-1337.

[20] Liu W., Sussman H.J., Shortest paths for sub-Riemannian metrics on rank-two distributions, Mem. Amer. Math. Soc. 118 (1995), no. 564, 104 pages.

[21] Montgomery R., A tour of subriemannian geometries, their geodesics and applications, Mathematical Surveys and Monographs, Vol. 91, American Mathematical Society, Providence, RI, 2002. 
[22] Nielsen M., Chuang I., Quantum computation and quantum information, Cambridge University Press, Cambridge, 2000.

[23] Respondek W., Feedback classification of nonlinear control systems on $\mathbf{R}^{2}$ and $\mathbf{R}^{3}$, in Geometry of Feedback and Optimal Control, Monogr. Textbooks Pure Appl. Math., Vol. 207, Dekker, New York, 1998, 347-381.

[24] Tall I.A., Respondek W., Feedback classification of nonlinear single-input control systems with controllable linearization: normal forms, canonical forms, and invariants, SIAM J. Control Optim. 41 (2002), 1498-1531.

[25] Wilkens G.R., Centro-affine geometry in the plane and feedback invariants of two-state scalar control systems, in Differential Geometry and Control (Boulder, CO, 1997), Proc. Sympos. Pure Math., Vol. 64, Amer. Math. Soc., Providence, RI, 1999, 319-333.

[26] Zhitomirskii M., Respondek W., Simple germs of corank one affine distributions, Singularities Symposium Łojasiewicz 70 (Kraków, 1996; Warsaw, 1996), Banach Center Publ., Vol. 44, Polish Acad. Sci., Warsaw, 1998, 269-276. 